\title{
THE USE OF OPTICAL COHERENCE TOMOGRAPHY ENVELOPE STATISTICS TO QUANTIFY LOCAL TEMPERATURE CHANGES IN TISSUE-MIMICKING PHANTOMS
}

\author{
by \\ Subaagari Seevaratnam \\ Bachelor of Engineering, Ryerson University, 2012 \\ A thesis \\ presented to Ryerson University \\ in partial fulfillment of the \\ requirements for the degree of \\ Master of Applied Science \\ in the Program of \\ Electrical and Computer Engineering
}

Toronto, Ontario, Canada, 2014

(C)Subaagari Seevaratnam 2014 


\section{AUTHOR'S DECLARATION FOR ELECTRONIC SUBMISSION OF A THESIS}

I hereby declare that I am the sole author of this thesis. This is a true copy of the thesis, including any required final revisions, as accepted by my examiners.

I authorize Ryerson University to lend this thesis to other institutions or individuals for the purpose of scholarly research.

I further authorize Ryerson University to reproduce this thesis by photocopying or by other means, in total or in part, at the request of other institutions or individuals for the purpose of scholarly research.

I understand that my thesis may be made electronically available to the public. 


\begin{abstract}
THE USE OF OPTICAL COHERENCE TOMOGRAPHY ENVELOPE STATISTICS TO QUANTIFY LOCAL TEMPERATURE CHANGES IN TISSUE-MIMICKING PHANTOMS
\end{abstract}

\author{
Subaagari Seevaratnam, B. Eng \\ Department of Electrical and Computer Engineering \\ Ryerson University, 2014
}

There exists a multitude of extreme thermally induced therapies for treating both benign and malignant tumors. Advancement in optics has shown prospect in clinical applications, especially monitoring oncological clinical treatments like thermal ablation. Optical coherence tomography (OCT) backscattered spectrum has demonstrated sensitivity to structural changes on the microscopic level. Envelope statistics analysis on OCT images of tissue-mimicking phantoms that are thermally modulated can provide structural information that correlates to changes in temperature.

Several probability distribution functions were analyzed by looking at suitable theoretical matches to the empirical OCT data. Results indicate that the generalized gamma function was the best fit and has potential in relating the scale parameter to the size of the scatterers in the phantom. Moreover, the parameters revealed sensitivity to temperature changes, which can be further studied to understand the biological response of tissue that are exposed to extreme thermal conditions in order to improve patient care. 


\section{Acknowledgment}

This two-year journey would not have been possible without some special people, who have truly made it a memorable one. I would like to extend my gratitude to:

- My supervisor, Dr. Beau A. Standish, for his support, encouragement and patient guidance through this project.

- My parents, for their unconditional love and support. Thank you dad for the numerous last minute drives to and from Kennedy Station; and thank you mom for morning chai, breakfast and the home cooked meals. Your cooking will always have a special place in my heart!

- My brother, Bardhi, who is more like a best friend. Thank you for the wise words of advice and keeping me in check with reality.

- Jamil and Ron, for being awesome lab mates (off paper) and friends. Thanks for the laughs, the helping hand and being awesome coffee mates with encouraging words. Moreover, thank you Jamil for pulling all-nighters to image phantoms and talk OCT concepts.

- Joel and Barry, who were always helpful with acquiring data, making all sorts of interesting phantoms, fixing the OCT system and being food buddies.

- Dr. Michael C. Kolios and Golnaz for your helpful insight on envelope statistics.

- Dr. Gu for kindly agreeing to make FBG sensors for my study and Dr. Victor Yang for allowing me to use the equipment in the BBL lab to acquire data for my thesis.

- Stephanie Iossifidis for unforgettable Friday night hangouts. Thank you for being an understanding friend and hearing me vent during stressful times.

- My undergraduate buddies (you know who you are). The random check-ins and meet ups were always a pleasure.

- Lastly, my defence committee, Drs. Gu, Umapathy and Smith, for taking the time out of your busy schedule and providing helpful feedback on my thesis.

"The only person who is educated is the one who has learned how to learn and change." - Carl Rogers 


\section{Contents}

1 Introduction $\quad 1$

1.1 Cancer Incidence \& Mortality Rates . . . . . . . . . . . . . . . . . . . . . . . . . .

1.2 Thermal Ablation Techniques . . . . . . . . . . . . . . . . 3

1.3 Thermal Tissue Monitoring Procedures . . . . . . . . . . . . . . . 8

1.4 Thesis Motivation: Optical Coherence Tomography Envelope Statistics for Measuring Localized Relative Temperature in Tissue-Mimicking Phantoms . . . . . . 10

1.5 Optical Coherence Tomography $(\mathrm{OCT}) \ldots \ldots \ldots$. . . . . . . . . . 10

1.6 Project Scope . . . . . . . . . . . . . . . . . . . . 16

1.7 Design Requirements . . . . . . . . . . . . . . . . . 17

2 Methodology - Optics 19

2.1 Optical Sensor: Fiber Bragg Grating (FBG) . . . . . . . . . . . . . . . . . 19

2.2 Envelope Statistics . . . . . . . . . . . . . . . . . 22

3 Temperature Controlled Optics Study with FBG 29

3.1 Experimental Procedure . . . . . . . . . . . . . . . . . . . . . . . 29

3.2 Results and Discussion: FBG Calibration and Probe Fabrication . . . . . . . . . 32

3.3 Chapter 3 Summary . . . . . . . . . . . . . . . . . . 35

4 OCT Envelope Statistics $\quad 37$

4.1 Experimental Procedure . . . . . . . . . . . . . . . . . . . 37

4.1.1 Hot Plate Calibration Process - Thermo Scientific ELED HP130910-33Q

Cimarec . . . . . . . . . . . . . . . . . 37

4.1.2 OCT-Thermal Imaging Setup with Tissue-Mimicking Phantoms . . . . . . 37

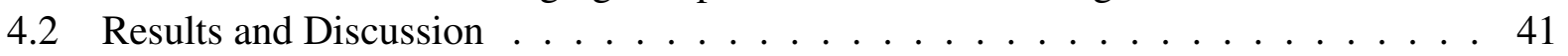

4.2.1 Hot Plate Calibration Curve . . . . . . . . . . . . . . . . 41

4.2.2 OCT Speckle and Envelope Statistics . . . . . . . . . . . . . . 43

4.3 Chapter 4 Summary . . . . . . . . . . . . . . . . . . . 54

5 Conclusion $\quad \mathbf{5 5}$

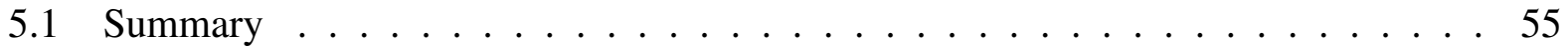


A Additional Work: Speckle Decorrelation

A.0.1 Speckle Decorrelation Measurements Potential to Quantify Local Temper-

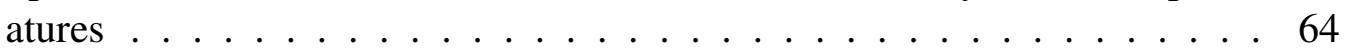

B Research Contributions 


\section{List of Figures}

1.1 The distribution of estimated new cases of cancer amongst the male and female population in Canada (2013) [1] . . . . . . . . . . . . . . . . . .

1.2 Example of the HIFU ablation procedure (part a) and the corresponding biological mechanism (part b), which intoduces pressure changes in cells. Pressure variations induce bubbles and causes changes in cell size, which eventually makes the cell collapse. During this event, the local energy released results in temperature elevation on the cellular level [4]. . . . . . . . . . . . . . . . . . . 4

1.3 Cellular response to cryotherapy [10]. a) Crystal formations cause direct injury, and produce a hypertonic (increased pressure) in the extracellular environment causing cell shrinkage. b) Vascular damage due to cold injury damages the endothelial cells; consequently, this causes clumping of platelets and leads to microthrombosis. Eventually, this leads to ischaemia, when an adequate volume doesnt reach the organ or tissue. c) Apoptosis (programmed cell death) is prompted by the reversible damage that occurs at the peripheral regions of the tumor volume.

1.4 A simplified schematic of a frequency domain OCT system [22]. The OCT primarily uses a Michelsons interferometer. A broadband light is split 50\% into the reference arm and 50\% in the sample arm, where the backscattered photons from both arms are measured at the detector. . . . . . . . . . . . . . . . 11

1.5 Microvasculature detection using speckle variance OCT imaging [26]. a) Dorsal skinfold window chamber model of a nude mouse. b) White light microscopy of the window chamber, which displays a close up of the general vicinity of the ROI. c) Fluorescence confocal imaging of the ROI $(1.8 \mathrm{~mm} \times 1.8 \mathrm{~mm}$ region of the window), after 10 min post the injection of the contrast agent. d) 3D speckle variance OCT image of the exact same ROI from the fluorescence confocal imaging, without the aid of any contrast agents. . . . . . . . . . . . . . . . . 14

1.6 Example of scattering interactions and the interference between the reflected light from the reference and sample arms of the OCT system [28] . . . . . . . . . . 15

1.7 The scope of the 'theradiagnostic' probe study, including the components of this thesis. . . . . . . . . . . . . . . . . . . . 16 
2.1 A single-mode optical fiber that contains a FBG and the corresponding spectrum graphs of the input, transmission and reflection spectrum [33]. The first graph (starting from the left) shows the spectrum of the broadband light source, the transmission spectrum is the signal measured once the light has propagated through the gratings and the reflected signal is the measurement of the single wavelength that has constructively interfered at each periodic grating. . . . . . . . . . . . 20

2.2 The parameters of the generalized gamma distribution for HFUS data for the diluted suspension samples of AML and PC-3 cells at different concentrations [44]. a) The shape parameter $a$ varies between the two solutions because of the difference in diameter size. b) The ratio $c / v$ increases with the number density, and considerably larger for PC-3 cells at higher concentration levels. . . . . . . . . . .

3.1 The FBG temperature study setup. Circulator (CIR); Fiber Bragg Grating (FBG); Thermocouple (TC); Multimeter (MM) . . . . . . . . . . . . . . . 30

3.2 Multi-luminal catheter's cross sections with dimensions. . . . . . . . . . . . . . 31

3.3 FBG thermal sensing catheter. This medical grade, multi-luminal catheter has the capability of housing five different functionalities. In this image, only one of the cavities is occupied with the FBG sensor (indicated by the dark blue region). The diameter of the catheter measures just under the $1.5 \mathrm{~mm}$ mark. . . . . . . . . . 32

3.4 The calibration curve (black line) along with results of the five trials [32]. The quantities of the five trials at each temperature increment exhibit that the results were within close proximity. The calibration curve is represented by the following equation: $b=0.010245 t+1290.2$ (equation 3.1) . . . . . . . . . . . . . .

4.1 The Schematic of the temperature measurement and OCT systems [49]; Circulator (CIR); Fiber Coupler (FC); Collimator (C); Balanced Detector (BD); Mirror (M); Polarization Controller (PC); Thermocouple (TC); Galvo Mirrors (GM); Lens (L); Multi-meter (MM); Polygon Mirror (PM); Semiconductor Optical Amplifier (SOA). 39

4.2 OCT image samples of: a) a titanium dioxide phantom that mimicks the refractive index of biological tissue and $b$ ) chicken tissue. . . . . . . . . . . . . 41

4.3 An example of an OCT image of the tissue-mimicking phantom with $3 \mathrm{~mm} \times 3 \mathrm{~mm}$ dimensions. The white granules in the image are referred to as speckle. . . . . . . 42

4.4 The calibration curve of the digital hot plate [49]. The calibration curve was configured based on the mean of all five trials. At each hot plate thermal setting, the standard deviation is represented with the error bars to indicate the variance in the thermocouple readings. . . . . . . . . . . . . . . . . . .

4.5 The Rayleigh, Rician, Generalized Gamma and Normal PDF fits of the envelope statistics of an OCT image of the titanium dioxide tissue mimicking phantom. . . . 44

4.6 The results of the three generalized gamma parameters for the titanium dioxide phantom with respect to modulating temperature [32]. . . . . . . . . . . . 45

4.7 The Rayleigh, Rician, Generalized Gamma and Normal PDF fits of the envelope statistics for the outline ROI of an OCT image of the tissue mimicking phantom. The blue dotted markers represents the empirical data. . . . . . . . . . . . 
4.8 A closer look at the Generalized Gamma fit to the experimental data, which was determined to by using the KS test. . . . . . . . . . . . . . . . . . . . 48

4.9 The Generalized Gamma distribution fits are shown above with respect to nine different temperature settings [49]. The changes with each peaks and position of the generalized gamma fits demonstrate that thermal changes influence the parameters that dictate the overall shape of the function. . . . . . . . . . . . . . 48

4.10 The three generalized gamma parameters observed within the range of $22^{\circ} \mathrm{C}-57^{\circ} \mathrm{C}$

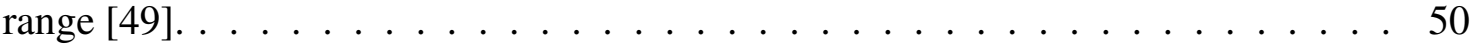

4.11 The line of best fit for the generalized gamma parameter $a$. The linear trend has a high correlation factor and is proven statistically significant with validation from the student-t test [49]. . . . . . . . . . . . . . . . . . . . . . . . . 52

A.1 Portrayal of the intensity fluctuation in the speckle and the corresponding time signal [49]. The dashed red box is the ROI, and the blue box is the pixel of interest. The time-dependent intensity signal looks similar to a fringe signal. . . . . . . . 65

A.2 The autocorrelation function of the intensity time signal at a sample temperature of $36 \mathrm{C}$ of a microsphere suspension fluid phantom [49]. . . . . . . . . . . . 67 


\section{List of Tables}

1.1 Summary of Thermal Ablation Methods . . . . . . . . . . . . . . . . 7

1.2 Comparative Table of Thermal Monitoring Methods. . . . . . . . . . . . . . . 9

3.1 Thermal Look-up Table based on FBG reading [32]. . . . . . . . . . . . . . . . . 34

3.2 Blind Test Results [32]; randomized hot plate temperature settings and resulting FBG measurements are shown above. Using equation 3.1 the temperature was determined by observing the Bragg wavelength readings from the spectrometer. . . 35

4.1 List of all the Optical and Electrical Components in the Thermal OCT Sensing System . . . . . . . . . . . . . . . . . . . . . . 40 40

4.2 The corresponding correlation factor for the four different PDFs of an OCT image, of the titanium dioxide phantom. . . . . . . . . . . . . . . . . . . 44

4.3 The corresponding correlation factor for the four different PDFs of an OCT image of the microsphere suspension phantom. . . . . . . . . . . . . . . . 46

4.4 The results of the two shape parameters $c$ and $v$, and the scale parameter $a$ with respect to changing temperature $[49] . \ldots \ldots \ldots 4 . \ldots \ldots$ 


\title{
Acronyms
}

\author{
AML: Acute Myeloid Leukemia \\ BBL: Biophotonics \& Bioengineering Laboratory \\ CT: Computed Tomography \\ DOCT: Doppler Optical Coherence Tomography \\ FBG: Fiber Bragg Grating \\ HIFU: High Intensity Focused Ultrasound \\ KS: Kolmogorov Smirnov (Test) \\ LITT: Laser Interstitial Thermal Therapy \\ MRI: Magnetic Resonance Imaging \\ OCT: Optical Coherence Tomography \\ OSA: Optical Spectrum Analyzer \\ PC: Prostate Adenocarcinoma \\ PDF: Probability Distribution Function \\ PDT: Photodynamic Therapy \\ RFA: Radiofrequency Ablation \\ ROI: Region of Interest \\ SS OCT: Swept Source Optical Coherence Tomography \\ US: Ultrasound \\ UV: Ultraviolet
}




\section{Chapter 1}

\section{Introduction}

DHIS introductory chapter provides an overview of relevant background information and the

main motivation of this thesis. It will give an insight into currently practiced thermal ablative therapies, thermal monitoring methodologies, the thesis motivation and hypothesis.

\subsection{Cancer Incidence \& Mortality Rates}

As of 2009, it has been estimated that roughly half the Canadian population will be diagnosed with cancer during their lifetime, equivalent to 2 in 5 Canadians [1]. The probability of females developing cancer is $41 \%$, whereas men rank slightly higher at $46 \%$ [1]. Approximately $25 \%$ succumb to this disease and Statistics Canada has acknowledged it as the leading cause of death amongst the Canadian population with a mortality rate of $29.8 \%$ (based on total deaths in 2009) [2]. Trends reveal that a majority of reported cancer cases will occur in the aging population. Between the time-period of 2012 to 2036, the demographics of seniors in the Canadian population will double and as such the cancer incidence and mortality rates will also grow substantially [1]. In 2013, it was estimated that 187,600 new incidences of cancer occurred consisted of breast, prostate, lung and colorectal cancer [1]. Figure 1.1 provides the distribution of the male and female new cancer case estimations for the year 2013. The incidence rates for the different types of cancer vary within the two genders, but the most prominent are prostate and breast cancer in men and women, respectively [3]. Based on the stats of the 187,600 new cases, prostate cancer will effect 1 in 7 males and breast cancer cases will effect 1 in 9 females during their lifetime [1]. 


\begin{tabular}{|c|c|c|c|}
\hline \multicolumn{2}{|l|}{$\begin{array}{l}\text { Males } \\
96,200 \\
\text { New cases }\end{array}$} & \multicolumn{2}{|l|}{$\begin{array}{l}\text { Females } \\
91,400 \\
\text { New cases }\end{array}$} \\
\hline Prostate & $24.5 \%$ & Breast & $26.1 \%$ \\
\hline Lung & $13.8 \%$ & Lung & $13.3 \%$ \\
\hline Colorectal & $13.8 \%$ & Colorectal & $11.6 \%$ \\
\hline Bladder & $6.1 \%$ & Body of uterus & $6.1 \%$ \\
\hline Non-Hodgkin lymphoma & $4.4 \%$ & Thyroid & $4.8 \%$ \\
\hline Kidney & $3.8 \%$ & Non-Hodgkin lymphoma & $3.9 \%$ \\
\hline Leukemia & $3.4 \%$ & Melanoma & $3.0 \%$ \\
\hline Melanoma & $3.4 \%$ & Ovary & $2.9 \%$ \\
\hline Oral & $2.9 \%$ & Leukemia & $2.7 \%$ \\
\hline Pancreas & $2.4 \%$ & Pancreas & $2.6 \%$ \\
\hline Stomach & $2.2 \%$ & Kidney & $2.5 \%$ \\
\hline Brain & $1.7 \%$ & Bladder & $2.2 \%$ \\
\hline Liver & $1.6 \%$ & Cervix & $1.6 \%$ \\
\hline Esophagus & $1.6 \%$ & Oral & $1.5 \%$ \\
\hline Multiple myeloma & $1.5 \%$ & Brain & $1.3 \%$ \\
\hline Thyroid & $1.3 \%$ & Stomach & $1.3 \%$ \\
\hline Testis & $1.0 \%$ & Multiple myeloma & $1.2 \%$ \\
\hline Larynx & $0.9 \%$ & Liver & $0.5 \%$ \\
\hline Hodgkin lymphoma & $0.6 \%$ & Esophagus & $0.5 \%$ \\
\hline Breast & $0.2 \%$ & Hodgkin lymphoma & $0.5 \%$ \\
\hline All other cancers & $8.9 \%$ & Larynx & $0.2 \%$ \\
\hline & & All other cancers & $9.6 \%$ \\
\hline
\end{tabular}

Figure 1.1: The distribution of estimated new cases of cancer amongst the male and female population in Canada (2013) [1] 
With the development of modern medical imaging, minimally invasive ablation of tumors has become a prominent form of therapy for patients, who do not qualify for conventional methods of treatment. There are distinct therapy options that are thermal or energy based, which are commonly used to ablate tumors of many types, such as breast and prostate tumors; some examples of currently practiced procedures include high intensity focused ultrasound (HIFU) [4, 5], radiofrequency ablation (RFA) [6,7], cryoablation [8] and laser interstitial thermal therapy (LITT) $[9,10]$. Thermal ablation utilizes either extreme high or low temperatures to induce coagulation and permanent cell injury where the primary aim of the treatment is to induce cell necrosis and/or tumor apoptosis [11]. The next section will further describe the various kinds of thermotherapies and their biological effects.

\subsection{Thermal Ablation Techniques}

Thermal ablation was developed during the 1990s, subsequent to the introduction of cross-sectional imaging, and has since been implemented as a substitute to conventional surgery of benign and malignant tumors [10]. Throughout the years, thermotherapy has gained significant popularity because it has reduced morbidity rates, increased conservation of healthy tissue, saved time and money spent for patient accommodations [3,4]. Prompting cell death can be implemented using hyper or hypothermic conditions, which results in thermal toxicity, protein denaturation, cell injury, vasculature damage and coagulative necrosis. However, these effects vary amongst the different types of modalities $[3,4]$.

HIFU is classified as the only non-invasive hyperthermic therapy that utilizes multiple focused ultrasound (US) beams on a region of interest (ROI) to produce cell lesions by increasing the temperature in the localized area up to $60^{\circ} \mathrm{C}[4,12]$. Figure 1.2a illustrates how this noninvasive method produces target ablation using an US beam. The concentration of high frequency US waves resonating at a frequency range of $0.8-3.5 \mathrm{MHz}$ generate acoustic cavitation, which result from the dilation and constriction of gaseous nuclei in cancerous cells, eliciting necrosis [5]. Figure $1.2 \mathrm{~b}$ demonstrates how the acoustic pressure from the US waves form bubbles, leading to 
cell death. Similar to HIFU, RFA and LITT are classified as hyperthermic therapy as these invasive procedures utilize electromagnetic energy to elevate tumor temperature.

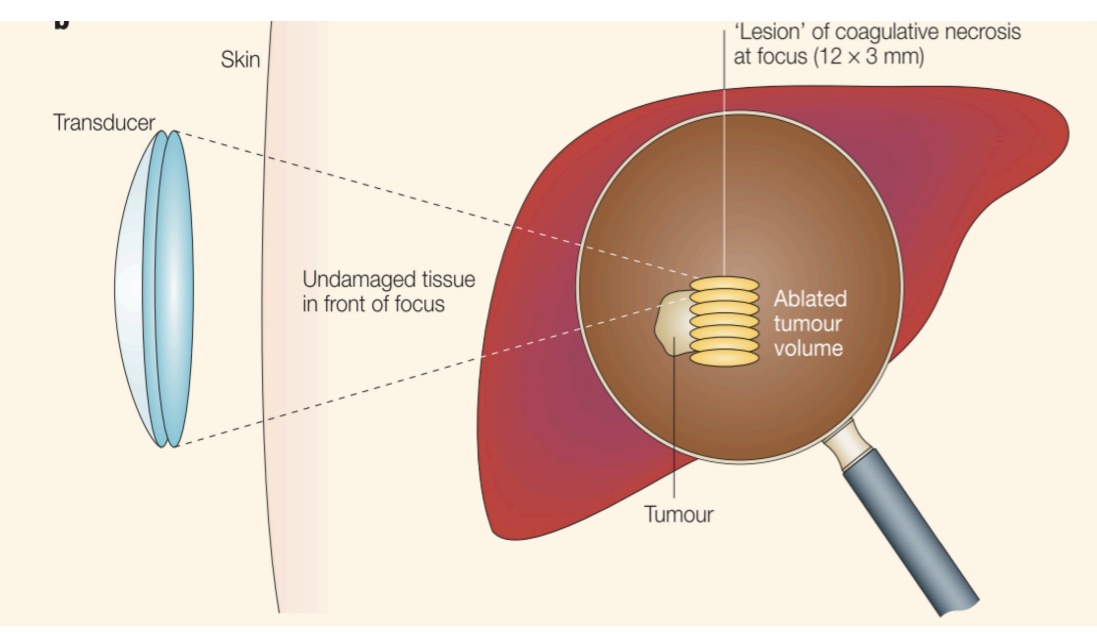

(a) Schmetic of multiple ablation lesions using HIFU

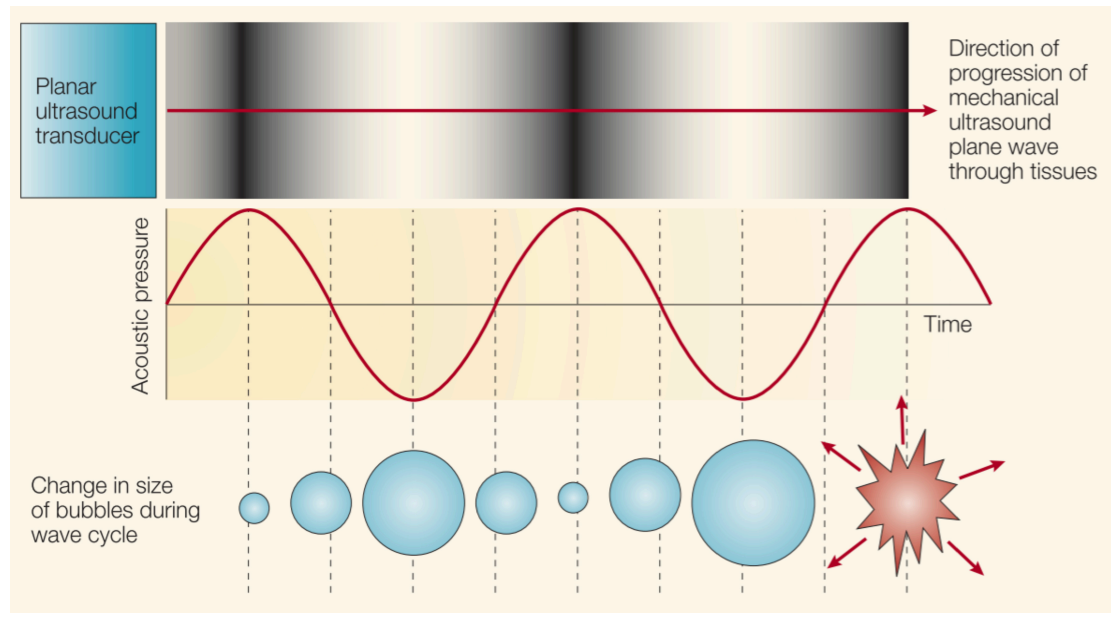

(b) Cavitation caused by HIFU

Figure 1.2: Example of the HIFU ablation procedure (part a) and the corresponding biological mechanism (part b), which intoduces pressure changes in cells. Pressure variations induce bubbles and causes changes in cell size, which eventually makes the cell collapse. During this event, the local energy released results in temperature elevation on the cellular level [4]. 
Percutaneous RFA administer one or more radiofrequency electrodes into the tumor with the aid of image-guidance (such as: US, computed tomography (CT) or magnetic resonance imaging $(\mathrm{MRI}))$ [6]. Temperatures ranging from $60^{\circ} \mathrm{C}$ to $100^{\circ} \mathrm{C}$ are generated by a high-frequency current, which initiates resistive heating [3,7]. The efficiency of RFA procedure is dependent on the localized temperature generated by the probes and requires the temperature to be maintained with the $60^{\circ} \mathrm{C}$ to $100^{\circ} \mathrm{C}$ range [10]. Previous, RFA clinical studies have observed that when the localized temperature exceeds $100^{\circ} \mathrm{C}$, the tissue experiences water vaporization and charring which increases tissue impedance, and in turn restricts electrical conduction [10]. Another limitation associated with RFA is the heat-sink effect, where tumors that are located within the proximity of vasculature reduce the effectiveness of the therapy due to heat dissipation from blood circulating away from the ROI [10]. These limitations in the RFA procedure impact the effectiveness of the therapy, which can be avoided with the aid of a temperature feedback mechanism. Comparably, LITT produces hyperthermic conditions by embedding radioactive isotope seeds within the tumor and elevating the temperature of the confined region as it is exposed to a certain wavelength of light [9]. The light exposure is introduced to the ROI via optical fibers, with the assistance of image guidance. The described therapy options all induce hyperthermic injury, which alters the biological environment by provoking membrane and organelle damages in cancerous cells. The ROI can be divided into two major regions: (1) the central zone and (2) the transitional zone [13].

The central zone is recognized as the area that has the first point of contact with the application tip and initiates an immediate coagulative necrosis effect, referred to as a direct mechanism [13]. The application tip can be either the tip of a radiofrequency electrode, tip of the LITT probe or the focal point of a high-frequency US beam. The transitional zone is the region peripheral to the central zone, and it experiences thermal conduction that radiates from the source and as a result contributes to cell apoptosis and/or permanent cellular damage [10]. Direct cellular damage is dependent on factors like the intensity of thermal energy, the frequency of application and the responsiveness of the tissue to the therapy [14]. When the local temperature of the tissue reaches the threshold of $40-45^{\circ} \mathrm{C}$ and has been exposed for an extended period of time, the cells undergo 
irreversible cell damage. When the temperature exceeds $60^{\circ} \mathrm{C}$ the proteins start to denature within the cell and a cytotoxic reaction commences resulting in coagulative necrosis.

On the contrary, hypothermic ablation is also capable of prompting cell death with a procedure named cryoablation. Compared to other energy-based ablation techniques, cryoablation has a long clinical history as it was first implemented in the 1840s to treat breast and uterine cancer. However, it acquired more attention during the 1960s as it was integrated with the development of probes that delivered liquid nitrogen [15]. Cryoablation uses the gas expansion mechanism with the aid of probes to reduce the temperature of probes distal tip to $-160^{\circ} \mathrm{C}$ as the gas evaporates $[10,14]$. The required temperature for cell fatality is within the range of $-20^{\circ} \mathrm{C}$ to $-40^{\circ} \mathrm{C}$, and to ensure the efficiency of the procedure the probe must maintain this temperature at least 1 $\mathrm{cm}$ beyond the tumors peripheral boundaries $[16,17]$. Cell injury occurs due to two main reasons: cellular dehydration and vasculature damage $[4,18]$. The effectiveness of cryoablation is reliant on the repetition of a freeze-thaw cycle. The formation of ice crystals on the extracellular space of the cell increases solute concentration, which produces hyperosmotic conditions [19]. As a result, the cell size reduces due to fluid efflux and induces alterations in the cellular membrane [10]. When the cycle enters the thaw phase, the intracellular pressure increases significantly and the corresponding fluid influx causes the cell to rupture [10]. Vasculature impairment occurs from deterioration of the endothelial layer and leads to platelet accumulation, decrease in blood flow and formation of blood clots [10]. Figure 1.3 outlines the mechanisms behind cell death during cryoablation. Clinical experiments from previous studies exhibit that cell death is guaranteed with a minimum of two freeze-thaw cycles at a temperature of at least $-40^{\circ} \mathrm{C}$ for a span of three minutes [19]. The ablative therapy methods (Table 1.1) mentioned above currently have no fixed method to monitor the thermal-biological effects of the treatment. This will be discussed further in the next section, which briefs on research conducted to establish a thermal metric to trace temperature. Table 1.1 summarizes the key characteristics of the previously mentioned ablation techniques. 


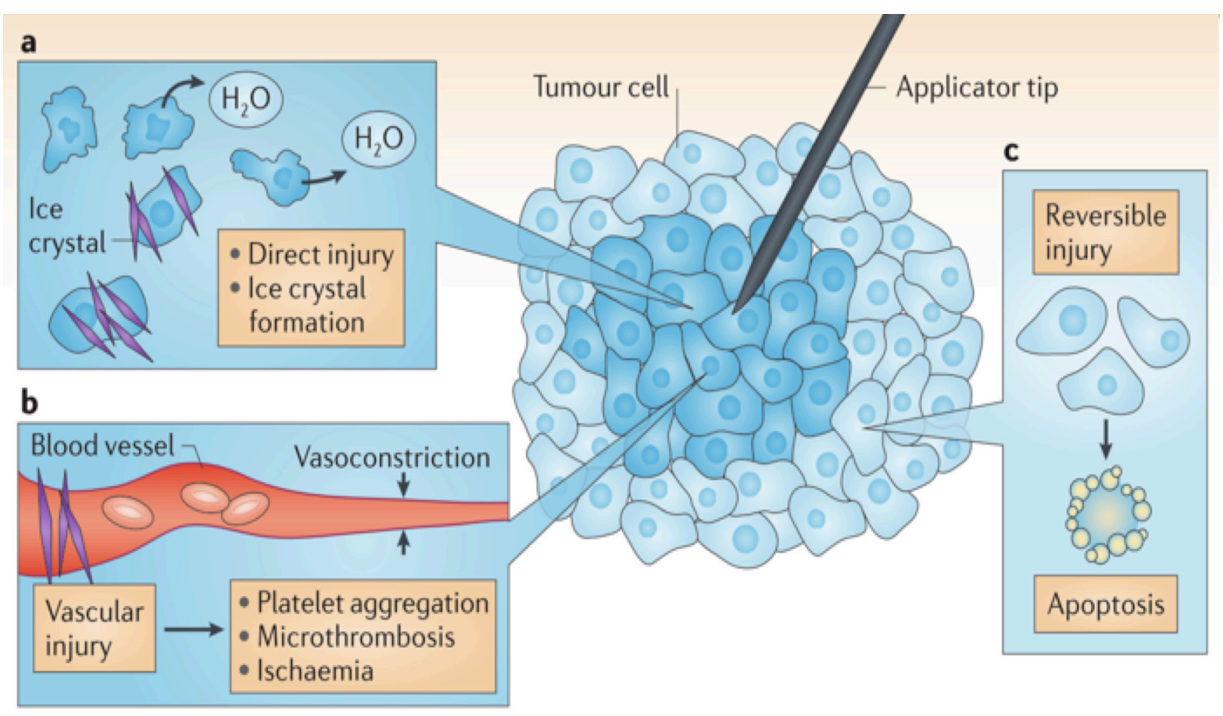

Figure 1.3: Cellular response to cryotherapy [10]. a) Crystal formations cause direct injury, and produce a hypertonic (increased pressure) in the extracellular environment causing cell shrinkage. b) Vascular damage due to cold injury damages the endothelial cells; consequently, this causes clumping of platelets and leads to microthrombosis. Eventually, this leads to ischaemia, when an adequate volume doesnt reach the organ or tissue. c) Apoptosis (programmed cell death) is prompted by the reversible damage that occurs at the peripheral regions of the tumor volume.

Table 1.1: Summary of Thermal Ablation Methods

\begin{tabular}{|c|c|c|c|c|}
\hline Characteristics & HIFU & RFA & LITT & Cryotherapy \\
\hline \hline Mechanism & Acoustics & Radiofrequency & Light & $\begin{array}{l}\text { Freeze-Thaw } \\
\text { Cycle }\end{array}$ \\
\hline $\begin{array}{l}\text { Hyper-/ } \\
\text { Hypothermic }\end{array}$ & Hyperthermic & Hyperthermic & Hyperthermic & Hypothermic \\
\hline Tumour Types & $\begin{array}{l}\text { liver, prostate, } \\
\text { bladder, kidney } \\
\text { and breast }\end{array}$ & $\begin{array}{l}\text { kidneys, breast, } \\
\text { bone and lungs }\end{array}$ & $\begin{array}{l}\text { breast, brain, } \\
\text { liver and } \\
\text { prostate }\end{array}$ & $\begin{array}{l}\text { retina, skin, } \\
\text { prostate, kidney, } \\
\text { liver, breast, } \\
\text { lung and bone }\end{array}$ \\
\hline
\end{tabular}




\subsection{Thermal Tissue Monitoring Procedures}

In order to advance the progression of thermal ablation therapies, it is vital to understand the biological effects post-therapy to improve treatment outcomes. Due to diverse tumor environments and varying thermal reactions, live temperature feedback during therapy could help to personalize and optimize therapies for patients. The biological correlation between thermal ablation and cancerous tissues can potentially augment survival rates, lower cancer recurrences and aid in efficiently targeting and ablating tumors. Currently, the previously mentioned ablation applications are implemented with little to no temperature sensing procedures and limited image guidance, which poses a significant problem as this gives clinicians no control and decreases the certainty on the aftermath of the ablation therapy. Lack of certitude or details on the biological outcomes that transpire during these procedures could possibly lead to the under-treatment or over-treatment of the region to be targeted. One key requirement for thermal ablative procedures is to regulate the extent of thermal injury in order to preserve the state of healthy tissue. For example, the anatomical location of the prostate is within a close proximity of vital organs, like the bladder, urethra and rectum. It is essential to protect the integrity of these biological structures because injury can affect the quality of life of the patient post-therapy, as it can reduce the functionality of the organs. Based

on these requirements, developing a thermal monitoring system that is minimally invasive and provides real-time temperature feedback can substantiate these thermotherapies to be successfully implemented for tumor ablation techniques. There are several proposed thermometry methods to monitor the local temperature of a target volume during thermo ablation, but these methods are still under extensive research to improve the accuracy of temperature measurements.

The most predominantly utilized method for detecting temperature are thermocouples, which provides a point-source thermal measurement with the site it is in contact with [20]. Due to this limitation, several probes are administered to get a better visualization of the thermal distribution in the ROI. Another predominantly studied modality to assess thermal distribution is the MRI, which has applied many techniques to analyze thermal changes [21]. The thermal information acquired using MRI techniques provide temperature gradients on a broader scale, which is inconvenient for 
a procedure that happens within a confined region. US parameters have also been thoroughly studied with respect to temperature transients resulting from ablation techniques. US parameters like speed of sound, the backscatter and attenuation properties of energy, are all susceptible to changes when the temperature of the medium has been modulated [20]. In cryoablation procedures, temperature measurements are acquired during a cryoablative procedure, and the speeds of the sound waves were analyzed during the freeze-thaw cycle. It was observed during crystal formulation that the speed of sound had significantly increased and decreased in the thawing session [20]. The accuracy of US thermal monitoring is always up for debate as the information can become difficult to interpret when bubbles start to form, which is a dominant feature in RFA [21]. Table 1.2 provides the features of the thermal monitoring methods and outlines the shortcomings of the each modality, which we aim to overcome in this thesis.

Table 1.2: Comparative Table of Thermal Monitoring Methods.

\begin{tabular}{|c||c|c|c|}
\hline Modality & Thermocouple & MRI & US \\
\hline \hline Imaging Capability & $\mathrm{x}$ & $\checkmark$ & $\checkmark$ \\
\hline Resolution & N/A & Low & Low \\
\hline Blood Flow Measurement & $\mathrm{x}$ & $\checkmark$ & $\checkmark$ \\
\hline Microvasculature Mapping & $\mathrm{x}$ & $\mathrm{x}$ & $\mathrm{x}$ \\
\hline Cost & Cheap & Expensive & Low \\
\hline
\end{tabular}




\subsection{Thesis Motivation: Optical Coherence Tomography Enve- lope Statistics for Measuring Localized Relative Tempera- ture in Tissue-Mimicking Phantoms}

Optical coherence tomography (OCT) has the potential to satisfy the requirements to make these thermotherapies successful in clinical applications, in comparison to other modalities (MRI, US and thermocouples). OCT has been extensively implemented in clinical trials and is established as a minimally invasive imaging modality, inexpensive, provides high-resolution imaging, blood flow detection, microvasculature mapping and capabilities of using a multiple probe apparatus to observe sensitive tissue regions within the vicinity of the target volume. All of these OCT features have been successfully utilized in monitoring the biological responses of tumors during some form of cancer therapy, and could potentially be implemented as an external feature simultaneously with the temperature metric. It is essential to acknowledge the fact that there are some drawbacks of using OCT, such as: limited perception of anatomical location due to restrictive penetration depth. However, thermal ablation is also constrained by the tumor size factor because lesions produced by many of these techniques are only capable of ablating regions that are smaller than $5 \mathrm{~cm}$. Therefore, OCT can potentially be incorporated with these ablative procedures, as they are only capable of analyzing ROIs that have small dimensions. The preliminary study of this thesis provides the essential algorithm to potentially quantify the temperature in a localized region, which could possibly solve the lack of thermometric information during thermo-ablative procedures.

My thesis hypothesis is "OCT can image and provide biological and thermal feedback metrics required to personalize and augment the outcomes of thermotherapies by studying OCT envelope statistics."

\subsection{Optical Coherence Tomography (OCT)}

Swept source OCT (SS-OCT) is an imaging modality that is capable of visualizing biological structures at the histological level ( 10 micron $[\mu \mathrm{m}])$. OCT is often compared to US, but in- 


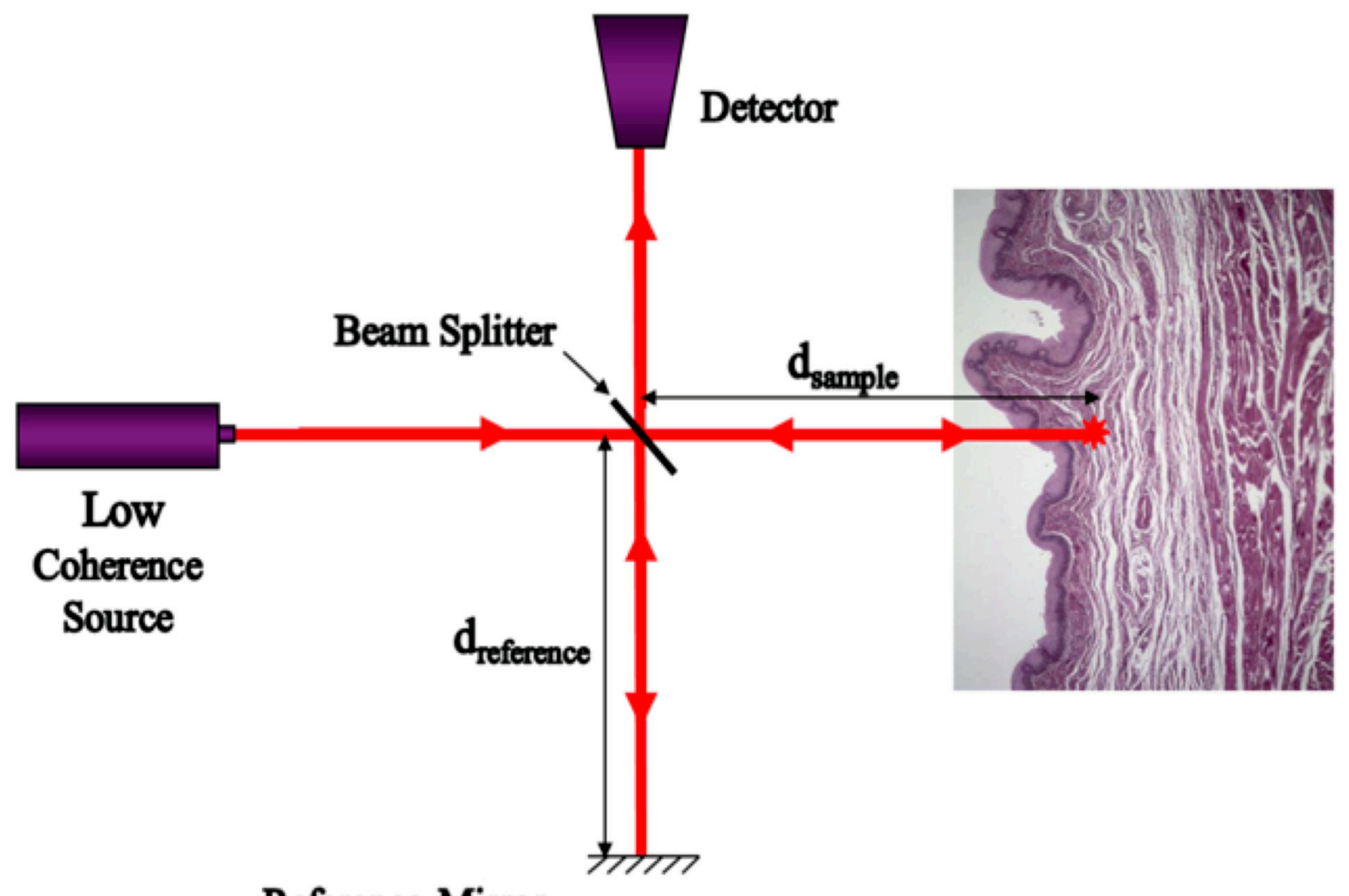

Reference Mirror

Figure 1.4: A simplified schematic of a frequency domain OCT system [22]. The OCT primarily uses a Michelsons interferometer. A broadband light is split 50\% into the reference arm and 50\% in the sample arm, where the backscattered photons from both arms are measured at the detector.

stead of quantifying backscattered pressure waves it measures backscattered photons. Measuring the local reflectivity of a sample using a SS-OCT system is feasible using an interferometer and a frequency based low coherent light source as shown in Figure 1.4. The light emitted from the source is equally divided into the reference arm and the sample arm using a beam splitter. The backscattered photons collected from both arms will either construct or deconstruct accordingly and will be quantified at the detector.

The local reflectivity of the sample is measured as function of depth, which makes up a single A-scan. Even though the OCT system provides high-resolution images, it can only image within 
a limited penetration depth that generally ranges between 1 to $3 \mathrm{~mm}$ [23]. The axial resolution of the OCT system is dependent on the coherence length of the light source, which is defined by the following equation 1.1:

$$
l_{c}=\frac{2 \times \ln 2}{\pi} \frac{\lambda_{c}^{2}}{\Delta \lambda}
$$

where, $l_{c}$ is the coherence length, $\lambda_{c}$ is the central wavelength and $\Delta \lambda$ is the spectral width of the light source (assuming a Gaussian spectrum) [24]. The transverse resolution of the OCT system is dependent on the numerical aperture and is defined by the following equation:

$$
\Delta x=\frac{4 \lambda_{c}}{\pi}\left(\frac{f}{d}\right)
$$

where, $\lambda_{c}$ is the central wavelength, $f$ is the focal length and $d$ is the lens diameter. The numerical aperture refers to the greatest angle at which a light can incident a lens so the light is totally internally reflected and is transmitted accordingly through the objective lens. Therefore, a low numerical aperture results in a low transverse resolution, and vice versa.

The A-scan contains the local reflection quantities within the range of the OCT systems coherence length, and anything beyond that threshold is information that cannot be seen because the system is no longer coherent. In order to acquire a 2D image (or a B-scan), either the sample arm or the sample must be translated laterally in a known pattern. A detailed description of the OCT system and its key characteristics are outlined in section 4.1.2.

Doppler OCT (DOCT) is implemented to detect motion of moving objects by analyzing shifts in Doppler frequency when the backscattered light from the moving object interferes with respect to the reference beam. DOCT is capable of providing structural images on the histological level and is commonly used to quantify blood flow and altering velocity in vasculature, which has been useful in detecting microvasculature changes during cancer therapies [25]. A study conducted by Standish et al. monitored vascular response with respect to photodynamic therapy (PDT) in real time, which provided biological feedback, therapy outcome control and information of the overall 
effectiveness of the treatment [23]. DOCT provides a contrast imaging metric for monitoring the termination of tumor necrosis by blood flow reduction rate, which is a demonstration of the various characteristics OCT possesses, like those listed in table 1.2 [23].

Mariampillai et al. research group has developed a successful interframe speckle variance imaging technique to detect microvasculature, based on the structural image intensity [26]. The algorithm takes the average of four adjacent A-scans, and improves the signal-to-noise ratio of the frame by reducing the speckle variance noise floor [26]. Speckle variance imaging was capable of perceiving vascular structures as small as $10 \mathrm{~m}$ in diameter [26]. This methodology is highly attractive for tracking treatments that target microvasculature changes, such as PDT. Figure 1.5 outlines the ROI and provides a visual comparison of the vasculature detection between fluorescence confocal microscopy and speckle variance OCT.

An inherent feature in OCT images is speckle, which can provide information about particle interactions within the sample. Speckle has been predominantly considered as noise in OCT images, however extensive research in this feature has established it as viable information for measurement procedures, such as determining size, shape and distribution without exclusively analyzing each particular particle in a sample [27]. Speckle patterns result due to the measurement of many wavelets from multiple backscattering sites within the coherent scattering region of volume.

The measured wavelets have random phases and amplitudes because they are scattering from different depths (within the coherence length), which can be represented by the following general expression:

$$
I_{d}=0.5\left(I_{r}+I_{s}^{\prime}\right)+\operatorname{Re}\left\{\left\langle E_{r}(t+\tau) E_{s}^{\prime}(t)\right\rangle\right\}
$$

where, $I_{d}$ is the intensity captured by the photodetector, $I_{r}$ and $I_{s}^{\prime}$ are the intensity (dc) reflected from the reference arm and the sample arm, respectively. $E_{r}$ and $E_{s}^{\prime}$ is the field of the reference arm and sample arm. The apostrophe indicates the modified intensity from the sample arm caused 


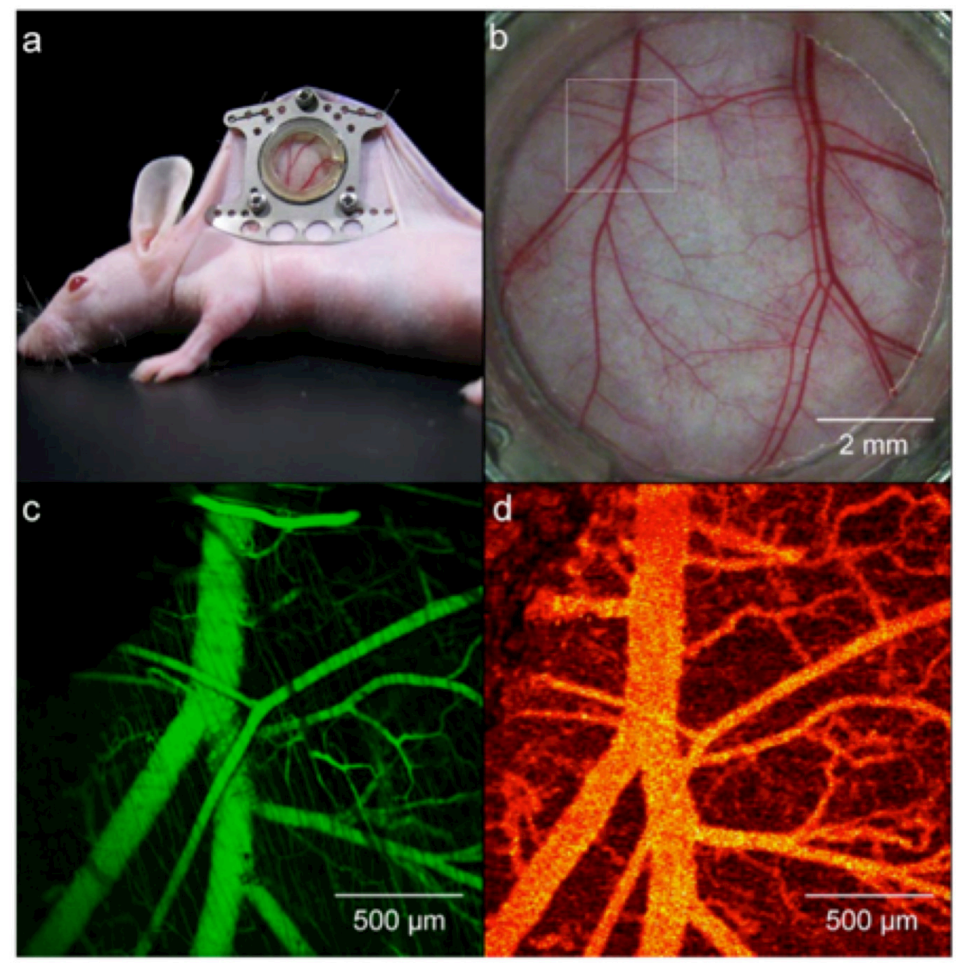

Figure 1.5: Microvasculature detection using speckle variance OCT imaging [26]. a) Dorsal skinfold window chamber model of a nude mouse. b) White light microscopy of the window chamber, which displays a close up of the general vicinity of the ROI. c) Fluorescence confocal imaging of the ROI ( $1.8 \mathrm{~mm} \times 1.8 \mathrm{~mm}$ region of the window), after $10 \mathrm{~min}$ post the injection of the contrast agent. d) 3D speckle variance OCT image of the exact same ROI from the fluorescence confocal imaging, without the aid of any contrast agents.

by the fluctuations in the refractive index at the backscatter sites. The detected intensity at the photodetector will exhibit variations in the phase and amplitude of the measured signal as indicated in equation 1.3. The $\tau$ is the optical time delay, which influences the phase of the signal when the signal is collected at the photodetector. Figure 1.6 illustrates the scattering interactions and the interference of the reference arm and modified sample arm signal at the photodetector. Speckle forms based on interference of the reflected signals from the two arms of the interferometer. 


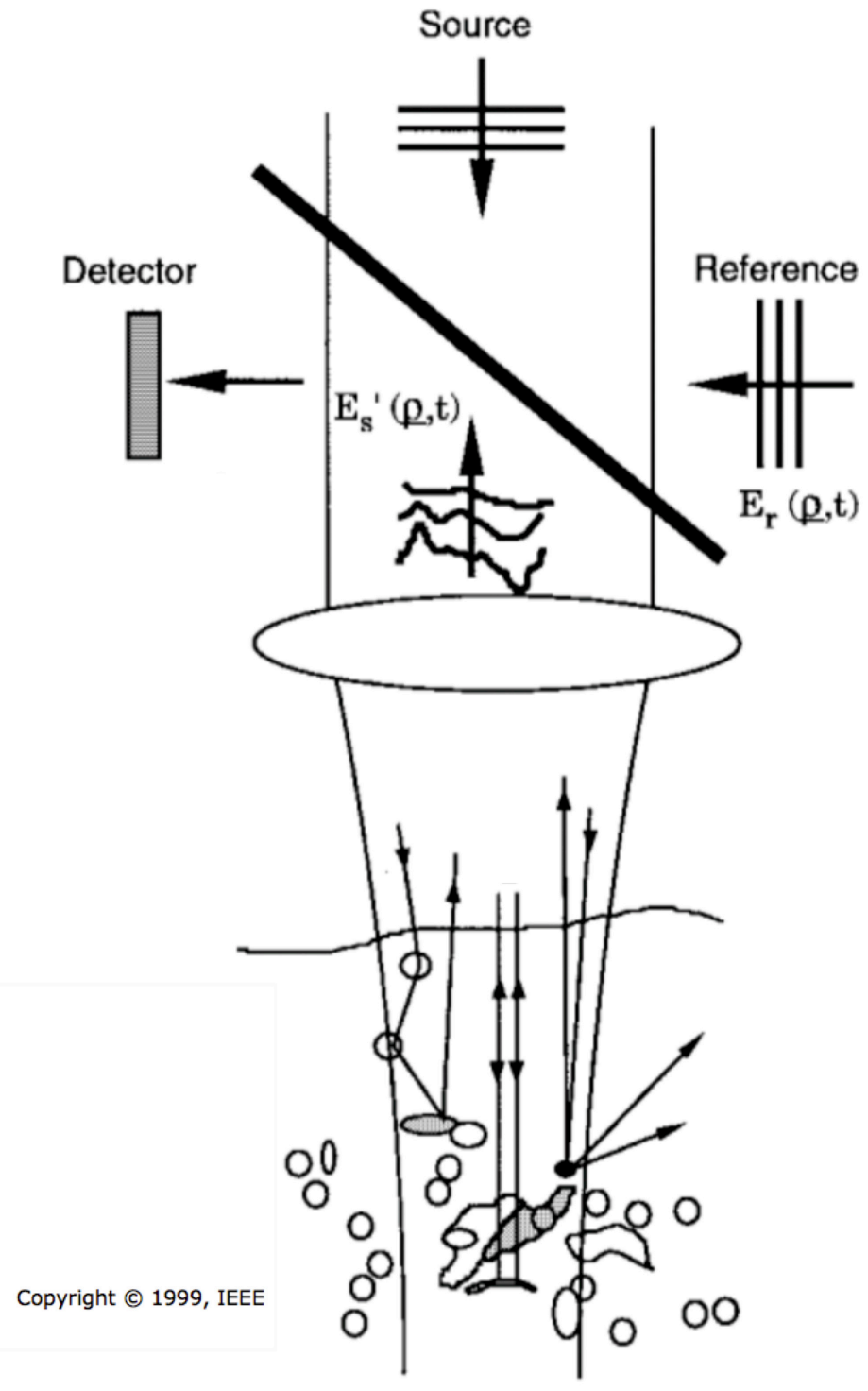

Figure 1.6: Example of scattering interactions and the interference between the reflected light from the reference and sample arms of the OCT system [28]. 
OCT has many established metrics, like those listed in Table 1.2. In this thesis, speckle characteristics were analyzed using envelope statistics, which can give insight on structural information of scatterer properties in a sample and can potentially provide an OCT contrast to analyze thermal conditions of cancerous tumors treated with thermal ablative procedures. The envelope statistics will be further discussed in the next chapter, which will explore the theory behind the distribution functions that were analyzed and research background on the application of envelope statistics used in HFUS and OCT.

\subsection{Project Scope}

The thesis starts by exploring the progression in optical sensors like fiber Bragg gratings (FBG), which has exhibited a promising potential in temperature probe studies. The integration of the probe with a statistical methodology to verify the local temperatures in tumor volumes can be a few of the many features of a multi-functional probe. The research conducted in this thesis is the partition of a bigger research project that aims to fabricate a multi-functional theradiagnostic probe, which has diagnosis and therapeutic functions. This Masters conducted, as my thesis comprises of the following components from the larger scope of the project, as seen in Figure 1.7:

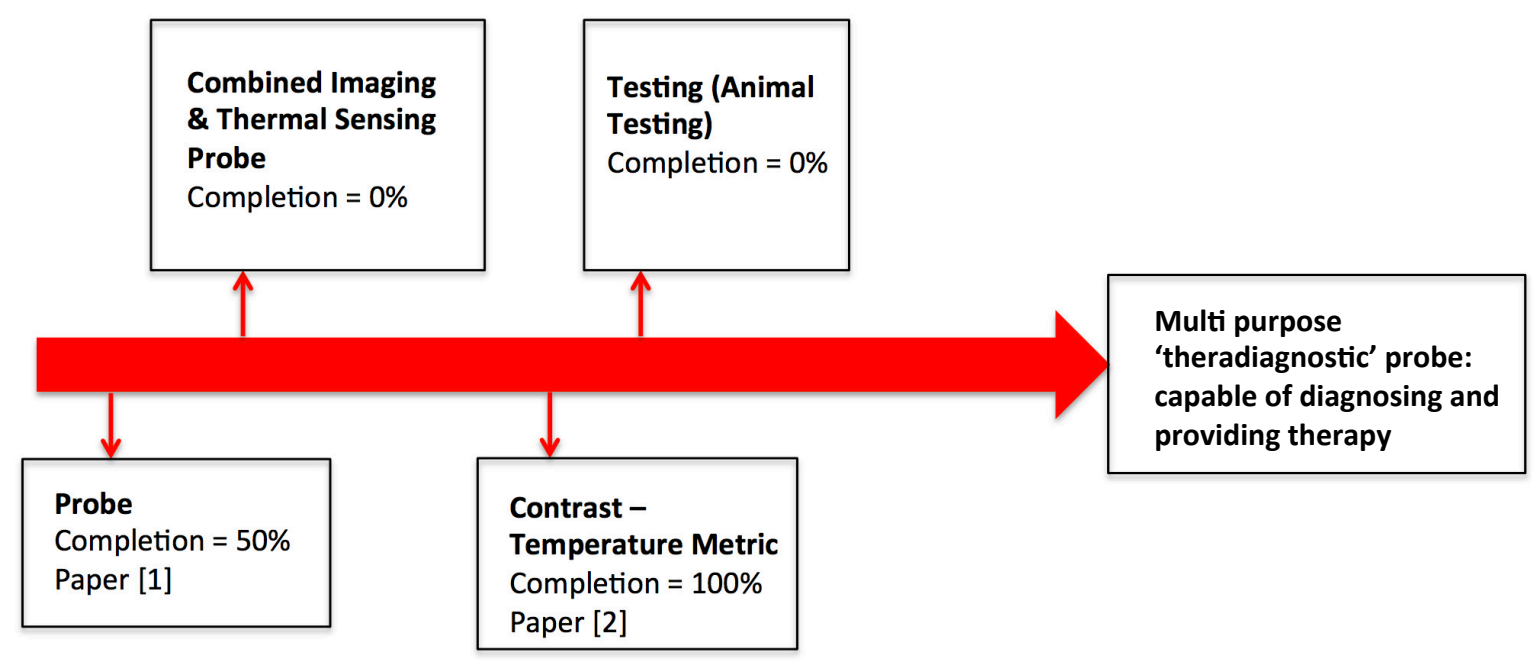

Figure 1.7: The scope of the 'theradiagnostic' probe study, including the components of this thesis. 
Research contributions that were published in conference proceedings, oral presentations and poster presentations can be found in Appendix B.

The motive behind studying temperature using FBG and OCT connects to the bigger notion of creating a theradiagnostic probe, which will have both therapeutic and diagnostic capabilities within one entity. The FBG will provide a single point thermal reading in the central zone, whereas the OCT will present thermal insight in the transitional zone of the tumor. This will provide clinicians information regarding thermal dosage, which will overall increase the efficiency of the thermal ablation procedure.

\subsection{Design Requirements}

There are certain design specifications that are mandatory for implementing vascular catheters and OCT imaging probes for clinical studies. Specific to this preliminary research for a temperature monitoring mechanism for thermal ablative procedures, there are some key considerations that need to be kept in mind through the design process:

1. Due to the study being in its initial phase, a phantom that mimics the optical properties of tissue is required to execute primary testing. Therefore, a phantom that matches the relative refractive index of tissue and tumors $(\sim 1.45-1.5)$ needs to be fabricated, that will produce images for a preliminary temperature-oriented study on speckle variations.

2. Develop an analysis of OCT images to assess speckle and structural changes in the sample (tissue-mimicking phantom) with respect to modulating temperature. This is the fundamental framework for obtaining thermal-relevant information from OCT images.

3. Calibrate the thermal readings from a FBG with a central wavelength of $1290.395 \mathrm{~nm}$ to gain preliminary knowledge to building a basic catheter apt for imaging tissue-mimicking phantoms and providing accurate feedback of the local temperature within the ROI. 
4. The sensor should not be interfered by MRI or US imaging modalities, which are the most commonly methods for thermal ablative procedures which require image-guidance.

5. Manufacture a multi-luminal probe that houses the thermal FBG sensor, and OCT imaging system; the catheter must have a diameter smaller than $1.5 \mathrm{~mm}$ to limit complications as clinical catheters are housed within a needle in order to penetrate the tissue. Moreover, the catheter design should be minimally invasive and should be easily integrated with the various ablation probes/catheters; hence it must be in the dimensional range of current catheters in the clinical setting. 


\section{Chapter 2}

\section{Methodology - Optics}

7 HE following chapter describes a literature survey of FBGs and OCT envelope statistics.

Understanding the fundamentals has helped to establish an experimental protocol, which was an iterative procedure that consisted of the experimental apparatus and the statistical algorithm for observing thermal variations in tissue-mimicking phantoms.

\subsection{Optical Sensor: Fiber Bragg Grating (FBG)}

FBG is an optical sensor that is constructed within a short segment of an optical fiber to sense metrics like temperature, strain and pressure. The apparent advantages of fiber optical sensors are:

1. Compact, light-weight, low power and are not vulnerable to electromagnetic interference [29]

2. High sensitivity [30]

3. Multiplex several sensors in an array (on a single fiber) [31]

4. Environmentally sturdy [29]

The FBG reflects a specific wavelength of light, via periodically varying the refractive index within the optical fiber core [32]. A broadband light source is connected to the optical fiber and at each grating a small reflection of a particular wavelength propagates back, which constructively interferes to form a single, large reflection. This is known as the Bragg reflection, as depicted in 
Figure 2.1.

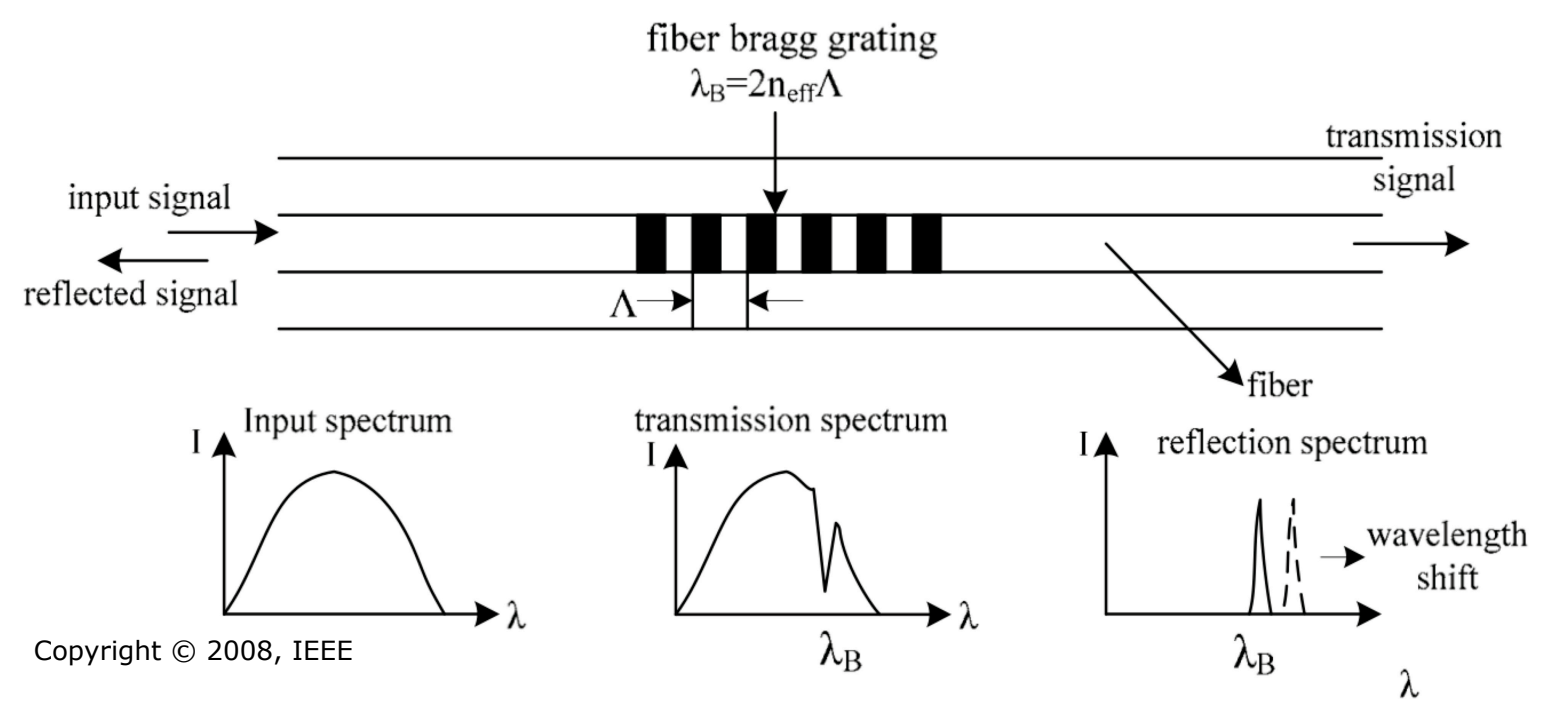

Figure 2.1: A single-mode optical fiber that contains a FBG and the corresponding spectrum graphs of the input, transmission and reflection spectrum [33]. The first graph (starting from the left) shows the spectrum of the broadband light source, the transmission spectrum is the signal measured once the light has propagated through the gratings and the reflected signal is the measurement of the single wavelength that has constructively interfered at each periodic grating.

The alternating refractive index pattern can be created, with the aid of two main components: ultraviolet (UV) light and a mask [34]. The optical core fiber is photosensitive where exposure to UV light will change the refractive index of the material [34]. The change in refractive index is dependent on the intensity of the UV light, the duration of exposure and the photosensitivity of the optical fiber [34]. The mask is crucial in order to attain the periodic pattern, as this will determine the particular Bragg wavelength that will be reflected.

The reflected wavelength is referred to as the Bragg wavelength and is defined by the following equation:

$$
\lambda_{b}=2 \times n_{e f f} \Lambda
$$


where, $n_{e f f}$ is the effective refractive index and $\Lambda$ is the grating period. The effective refractive index $\left(n_{e f f}\right)$ defines the velocity of the propagating light in comparison to its velocity in a vacuum [30-33]. The Bragg wavelength is susceptible to either increase or decrease with respect to changes in temperature or strain applied on the grating. FBGs follow the Snells Law that describes the relationship between the angle of incidence and refraction when a ray of light passes through a boundary of two different mediums. The difference in the Bragg wavelength, induced by temperature and/or strain, compared to the original Bragg wavelength is given with the following relationship:

$$
\frac{\Delta \lambda_{b}}{\lambda_{b}}=\left(1-p_{e}\right) \epsilon+\left(\alpha_{\Lambda}+\alpha_{\Pi}\right) \Delta T
$$

where, $\Delta \lambda_{b}$ is the shift in wavelength, $p_{e}$ is the strain-optic coefficient, $\epsilon$ is the strain, $\alpha_{\Lambda}$ is the thermal expansion coefficient, $\alpha_{\Pi}$ is the thermo-optic coefficient and $\Delta T$ is the change in temperature [30-33]. For a single mode fiber that contains a silica core doped in $\mathrm{GeO} 2$, the thermal expansion coefficient is approximately $0.55 \times 10-6\left[{ }^{\circ} \mathrm{C}\right]^{-1}[31,35]$, the thermal optic coefficient of $8.6 \times 10-6\left[{ }^{\circ} \mathrm{C}\right]^{-1}[31,35]$. When the grating is exposed to any change in temperature and/or strain, the grating spacing and the index of refraction is temporarily altered, until the environment is at room temperature and strain-less. The resulting changes in the Bragg wavelength occur due to mechanical stress or temperature being applied to the grating, as clearly shown by the coefficients that influence equation 2.2. The strain sensitivity of the grating is influenced by changes in grating spacing and refractive index due to the photoelastic effect (stress distribution) [31]. The thermal components of equation 2.2 have two main influencers: (1) The thermal expansion coefficient, which exhibits the rate of change of the optical fiber material (silica) in terms of volume with respect to modulating temperature and (2) the thermo-optic coefficient, which reveals the rate of change in the refractive index with respect to change in temperature [31]. In other words, changes resulting in the refractive index of the period of the gratings will shift the Bragg wavelength; however it is important to note that it is mainly caused by changes in the refractive index. The Bragg wavelength can be measured with the aid of an optical spectrum analyzer or a wavelength interrogator. The rate of change in wavelength with respect to temperature depends on the 
Bragg wavelength written in the fiber. For wavelengths close to $830 \mathrm{~nm}$ the rate of change is $\sim 6.8$ $\mathrm{pm} /{ }^{\circ} \mathrm{C}$, close to $1300 \mathrm{~nm}$ the rate of change is $\sim 10 \mathrm{pm} /{ }^{\circ} \mathrm{C}$, and closer to $1500 \mathrm{~nm}$ the rate of change is $\sim 13 \mathrm{pm} /{ }^{\circ} \mathrm{C}[36]$. FBGs have predominantly used in the industry for temperature sensing and temperature compensation within a nominal range because as soon as it is exposed to a grade of $500{ }^{\circ} \mathrm{C}$ or higher, the gratings will be damaged in the optical fiber [37].

Using FBGs for thermal ablative procedures has a series of benefits, which makes it highly appealing for clinical procedures. The Bragg wavelength shift experienced by the sensor is a linear response that is sustained for a large range of the measuring quantity. Second, the change in measurand is expressed in terms of wavelength; therefore the sensor signal isnt susceptible to discrepancies due to environmental noise or power loss [29]. Lastly, due to insignificant weight and small diameter, it poses little limitation for integration with medical grade catheters.

\subsection{Envelope Statistics}

Theoretical clinical research has been conducted in the past decade with respect to high frequency US envelope statistics and scatterer properties, which have demonstrated promising results in distinguishing properties like scatterer cross-section and effective scatterer number density [38]. U1trasonic backscattering events caused by tissue have been represented using the Gaussian model with the assumption that the scatterers within the medium are relatively within the same size range. Previous studies have used Rayleigh distribution to distinguish normal breast, liver and heart tissue [39]. Furthermore, the Rician model has been successfully implemented in identifying damaged heart tissue, due to some form of heart ailment [40]. A research team from Drexel University located at Philadelphia, PA generated the theoretical foundation between envelope statistics and scatterer properties in tissue [38,39]. Shankar et al. from Drexel University have conducted numerous studies, which were able to characterize cancerous breast tissue and differentiate between benign and malignant tumors using non-Rayleigh distribution, such as the $\mathrm{K}$ and Nakagami distribution to describe the backscatter echo from tissue [41, 42]. Expanding more on the HFUS envelope statistics, Raju et al. have successfully demonstrated that extending the same technique 
to higher frequencies can allow one to distinguish amidst different types of the skin layers [43].

High frequency US is highly responsive to structural changes experienced by scatterers, which makes this highly applicable for monitoring therapeutic outcomes. Assessment protocols for cancer treatments have patients receive a full treatment regiment before examining the progress of the treatment results with respect to tumor size or cellular alterations [44]. Tunis et al., has used envelope statistics to quantify structural changes that transpire during apoptosis and necrosis, which has provided a viable solution to monitoring tumor response during treatment in a non-invasive procedure [44]. The study analyzed the statistical parameters of the Generalized Gamma model for two diluted suspension samples with different sized cell lines, which provided information on cell density and the size of cells. The acute myeloid leukemia (AML) cells have a mean diameter of $12.5 \mathrm{~m}$ and the prostate adenocarcinoma (PC-3) cells have a diameter of $30 \mathrm{~m}$. Due to the PC-3 cells being larger in size, the AML cell solution required a higher number of cells per volume in order to attain the same volumetric concentration [44]. The scale parameter $a$ of the generalized gamma distribution represented the effective scatterer cross-section; the results exhibited an increase with respect to incrementing number density [44], as seen in Figure 2.2a. In the domain where the number density of the two solutions overlaps, the scale parameter $a$ is larger for PC-3 cells [44]. This is consistent with the theory that parameter $a$ reflects the cross-section of the scatterer. The diameter of the PC-3 cells is approximately twice the size of the AML cells, and a larger scale parameter is expected. The ratio of the two shape parameters $c / v$ reflects the number density of the solution [44]. Figure 2.2b exhibits the positive linear trend between the increasing number density of the solutions and the shape parameter ratio [44]. In the region of the overlap of the number density, the shape parameter ratio is equivalent between the two solutions. This indicates that the parameter is capable of reflecting changes in scatterer number density independent of the scatterer size [38]. The results agree with Shankar et al. proposition that the $c / v$ correlates to the effective scatterer number density [45]. 


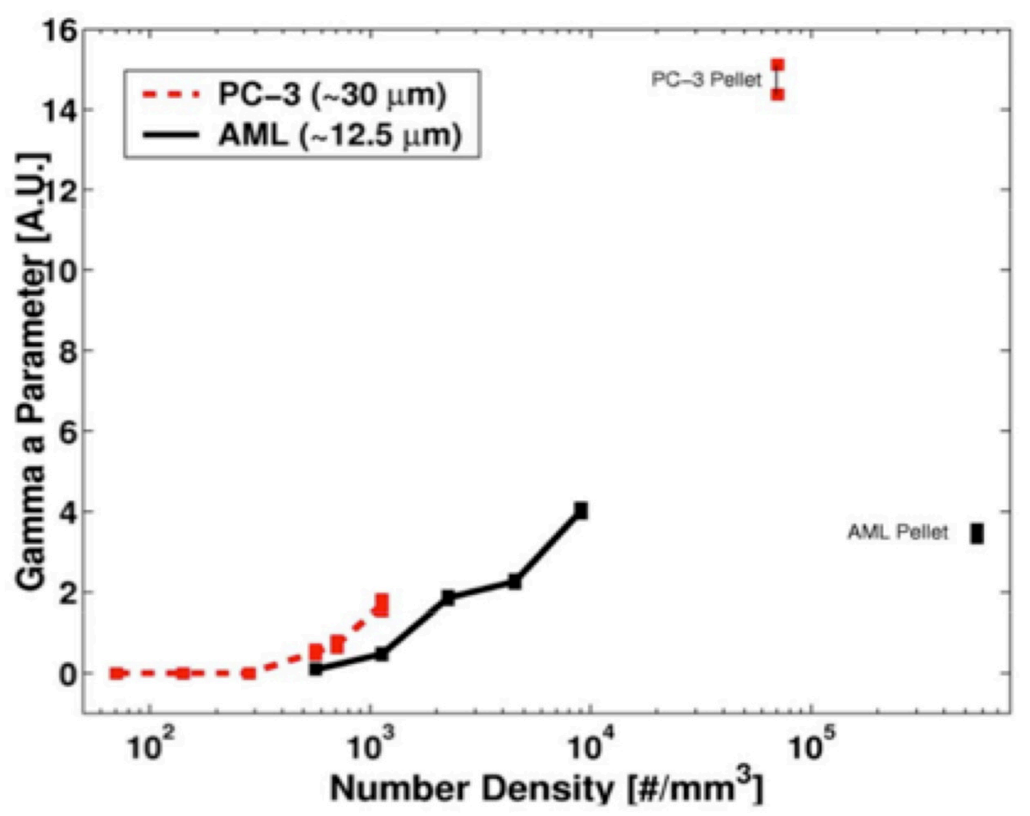

(a) The scale parameter $a$

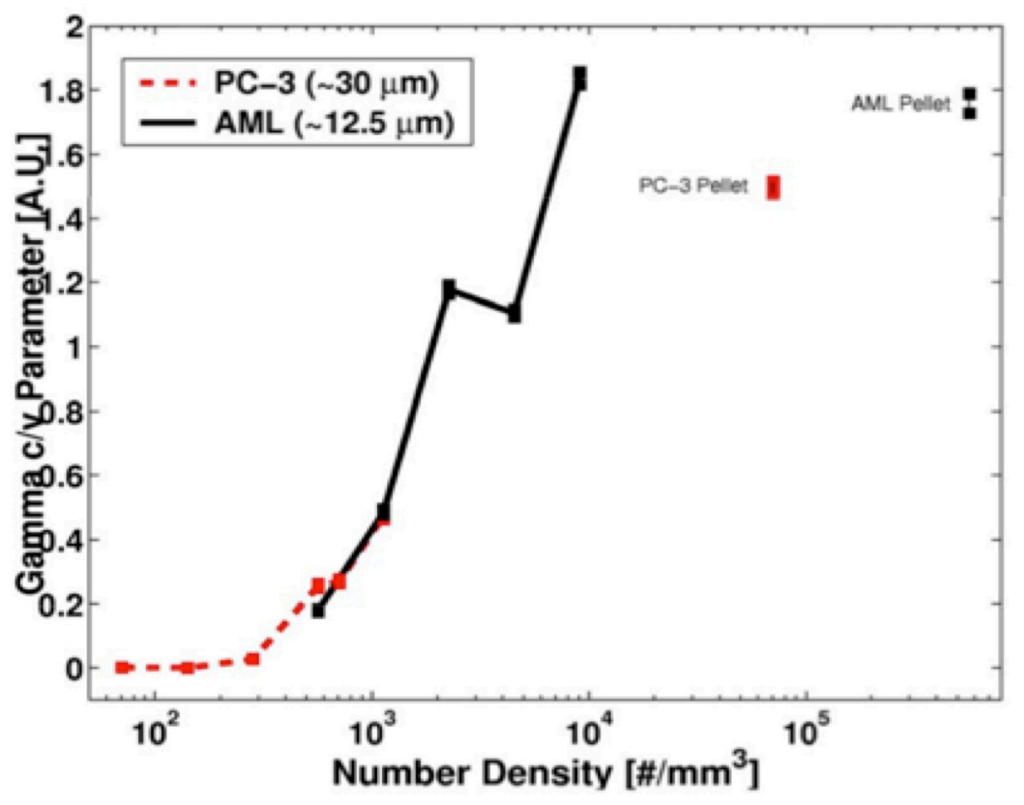

(b) . The shape parameter ratio $c / v$

Figure 2.2: The parameters of the generalized gamma distribution for HFUS data for the diluted suspension samples of AML and PC-3 cells at different concentrations [44]. a) The shape parameter $a$ varies between the two solutions because of the difference in diameter size. b) The ratio $c / v$ increases with the number density, and considerably larger for PC-3 cells at higher concentration levels. 
As previously mentioned, OCT is analogous to US in terms of data acquisition and the format of configuring an image. Based on these similarities, the fundamental theories that have been extensively studied in US can be applied in OCT. Previous research has shown variations in optical properties during cell death from therapy, which established that OCT can be a viable option for detecting these changes [27]. Prior to OCT, light scattering studies had an extensive background in oncological research. Techniques like light scattering spectroscopy have been used for in vivo studies to diagnose dysplasia by determining the nuclear size distribution. Golnaz et al. research has measured OCT backscattered signals to detect cell death by looking at structural changes during apoptosis with the envelope statistics methodology [27]. During the process of cell death, the backscattered intensity signals increased during the apoptosis and mitotic arrest phase of cell death and then a significant plunge in the intensity of the signal during necrosis.

The interferemetric nature of the OCT system produces a speckle pattern in its images. This is observed by the varying intensities of the pixels throughout the image. The light intensity measured from these multiple scatterers shares a similar spectral shape as the spectrum of the light source of the OCT system, and it is expected to resemble the shape of a Gaussian beam. Similar to US imaging, the OCT image is a resultant of the envelope of the signal. Therefore, the backscatter intensity also has an envelope with a particular statistical distribution, which modulates according to changes in the scatterer properties. The mechanical changes that the scatterers experience due to temperature modulations can potentially be represented quantitatively with envelope statistics.

The OCT speckle histogram was comparatively fitted to four different types of probability distribution functions (PDF), which were: Normal distribution, Rayleigh distribution, Generalized Gamma distribution and Rician distribution. The Normal distribution, also referred to as the Gaussian distribution, is a distribution function where the highest probability value is positioned at the median value of the sample data set [32]. The probability of the pixel intensities $(x)$ in a Normal distribution function are defined by the mean $(\mu)$ and the standard deviation $(\sigma)$ of the data set and is represented in the following form [32]: 


$$
f(x)=\frac{1}{\sigma \sqrt{2 \pi}} e^{-\frac{1}{2}\left(\frac{x-\mu}{\sigma}\right)^{2}}
$$

The Rayleigh distribution function is represented as:

$$
f(x)=\frac{x}{\alpha^{2}} e^{-\frac{x^{2}}{2 \alpha^{2}}}
$$

where $x$ is the pixel intensity and $\alpha$ is a scale parameter, which must be a real number [38]. Rayleigh distribution is perceptible only when the magnitudes of pixel intensities (that consists of two components: real and imaginary) are normally distributed, have the same variance with zero mean and are uncorrelated [38]. The Rician distribution, which is defined by the following equation:

$$
f(x \mid A)=\frac{x}{\sigma^{2}} e^{-\left(\frac{x^{2}+A^{2}}{2 \sigma^{2}}\right)} I_{o}\left(\frac{A x}{\sigma^{2}}\right) u(x)
$$

where, $I_{o}$ is the zeroth-order Bessel function, $x$ is the magnitude of each pixel intensity, $\sigma$ is the standard deviation and the $A$ is the magnitude of the real and complex quantities of the select pixels within a ROI, which is represented by this relationship [46]:

$$
A=\sqrt{A_{R}^{2}+A_{I}^{2}}
$$

The unit step in equation 2.5 is to ensure that the PDF expresses the positive values of the pixel intensities in the OCT image [46]. In the special case, if $A$ were to equal zero, the Rician distribution is equivalent to the Rayleigh PDF as seen in equation 2.3.

The generalized gamma distribution function is a PDF where it illustrates a higher probability placement within the first half of pixel intensity and steep descend in the second half, portraying a peak closer to the left end of the distribution. The generalized gamma distribution is represented as:

$$
f(x)=\frac{c x^{c v-1}}{a^{c v} \Gamma(v)} e^{-\left(\frac{x}{a}\right)^{c}}
$$


where $x$ is the pixel intensity, $a$ is a scale parameter, and $v$ and $c$ are shape parameters. $\Gamma(\cdot)$ represents the gamma function $[38,47]$. The Gamma distribution is a distinct variation of the Generalized gamma distribution when the shape parameter c is set to one [47]. As a result, the PDF for the Gamma distribution in a simplified form is seen below:

$$
f(x)=\frac{x^{v-1}}{a^{v} \Gamma(v)} e^{-\left(\frac{x}{a}\right)}
$$

When differentiating amongst the various PDFs, the Generalized gamma distribution is considerably more versatile in representing a sample set because there are more parameters to employ to fit the speckle envelope statistics [37]. The generalization of the PDF is accomplished by including the shape parameter $c$ in the exponential factor of the gamma distribution component as seen in equation 2.7 [47]. Not only is the generalized gamma PDF competent at adapting to a larger domain of scattering properties, but represents many other PDFs. When the two shape parameters $\mathrm{v}$ and $\mathrm{c}$ are equal to 1 and 2, respectively, the PDF simplifies to the Rayleigh distribution [44].

In order to determine the goodness of fit between the empirical data and the distribution functions of interest, the Kolmogorov-Smirnov (KS) test was applied. The KS test is a non-parametric test that compares a single dimensional probability distribution to a reference PDF, or compares two sample distributions to one another to determine if they originate from the same distribution family [32]. The test relies on the notion that the empirical distribution is asymptotic, in other words, the distribution must verge to zero as it approaches infinity. The goodness of fit is determined by observing the proximity of the cumulative frequency of the empirical distribution and the cumulative density function of the hypothetical data [43]. The largest measured distance between the observed and expected cumulative frequencies for the various PDFs is the qualifying factor to determine the best fit [48]. Therefore, the smallest of the measured distances amongst the PDFs would be determined as the best fit, which is represented with a correlation factor, $p$. The null hypothesis that an OCT envelope signal is a member of a particular PDF will be accepted if the correlation factor is less than a value of $0.05(\mathbf{p}<\mathbf{0 . 0 5})$ [48]. 
The speckle from the structural OCT images were categorized into bins based on their intensities, which created histograms that represented the count for every intensity available within the chosen ROI of the sample. The histogram of the empirical data was then compared to the four chosen PDFs: Normal distribution, Rayleigh distribution, Gamma distribution and Rician distribution. The histogram consisted of pixel intensity counts with the ROI, which were categorized and fit with the PDFs mentioned previously. The best fit was determined based on the correlation factor that is provided through the KS test. 


\section{Chapter 3}

\section{Temperature Controlled Optics Study with FBG}

HIS section outlines the groundwork for calibrating and assembling an elementary thermal
sensing probe using the FBG sensor.

\subsection{Experimental Procedure}

A preliminary study was conducted with an FBG sensor, written on a single mode fiber, to measure the changes in wavelength with respect to temperature with no stress/strain effects. An FBG sensor with a Bragg wavelength of $1290.395 \mathrm{~nm}$ was fabricated, and its resulting shifts were measured using an ISBEN, I-MON E series spectrometer that detected near-infrared light within the range of 1275 - $1345 \mathrm{~nm}$. The central Bragg wavelength was chosen to be $1290.395 \mathrm{~nm}$ because for future integration of the thermal-imaging system the system will only require one light source for FBG sensing and OCT imaging. This decreases the complexity and increases the compactness of the system. The FBG sensor had a reflectivity of 0.95 and the bandwidth of $0.2 \mathrm{~nm}$. An AFC broadband light source with a central wavelength of $1310 \mathrm{~nm}$, the FBG sensor and the spectrometer were interconnected through a circulator, which is a three-port device that is designed in such a manner that light entering a specific port will exit in the next adjacent port. In other words, the light that enters port 1 will channel out through port 2; if light were to be reflected back in through port 2 , the light will not migrate through port 1 , but will circulate to port 3 . The temperature measured via FBG with a corresponding wavelength was compared to the readings of a thermocouple. The 
physical setup of this experiment is shown in Figure 3.1.

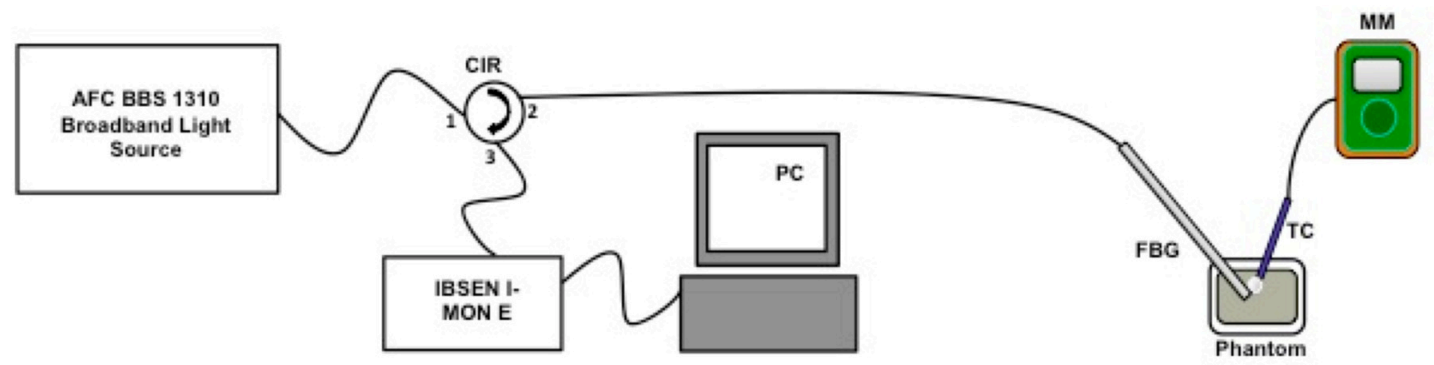

Figure 3.1: The FBG temperature study setup. Circulator (CIR); Fiber Bragg Grating (FBG); Thermocouple (TC); Multimeter (MM).

Based on this setup, five temperature-controlled trials were conducted to observe the Bragg wavelength shifts with little to no effects from strain. The FBG, which was submerged within the phantom, was heated to various temperatures using a digital hotplate (Corning 420D Hotplate). The resulting Bragg wavelength shifts were recorded between the temperature range of $25^{\circ} \mathrm{C}$ $60^{\circ} \mathrm{C}$ at $5^{\circ} \mathrm{C}$ increments. The results of the five trials were averaged, which produced a line of best fit and was used as a reference for any FBG thermal readings. The temperature setting of the fiber Bragg sensor was measured with reference to a thermocouple. In order to validate that the measurement of one thermocouple provided the most accurate results, multiple thermocouples were administered to verify that the measurand of the thermocouples were within close proximity. This procedure was to limit any potential errors in calibrating the FBG sensor with respect to temperature drifts.

The next step was to integrate the FBG sensor into a multi-luminal catheter, which contained five luminals that could serve different functions. Figure 3.2 is the cross-sectional illustration of the multi-luminal catheter, which would house FBG sensors in the peripheral luminals and the central luminal would house the OCT sample arm fiber. The diameter of the multi-luminal catheter is approximately $0.275 \mathrm{~mm}$, which was enough space to accomodate the FBG sensor that has a width of $250 \mu \mathrm{m}$ and leave space for fiber expansion. As mentioned in equation 2.2, the FBG sensor 


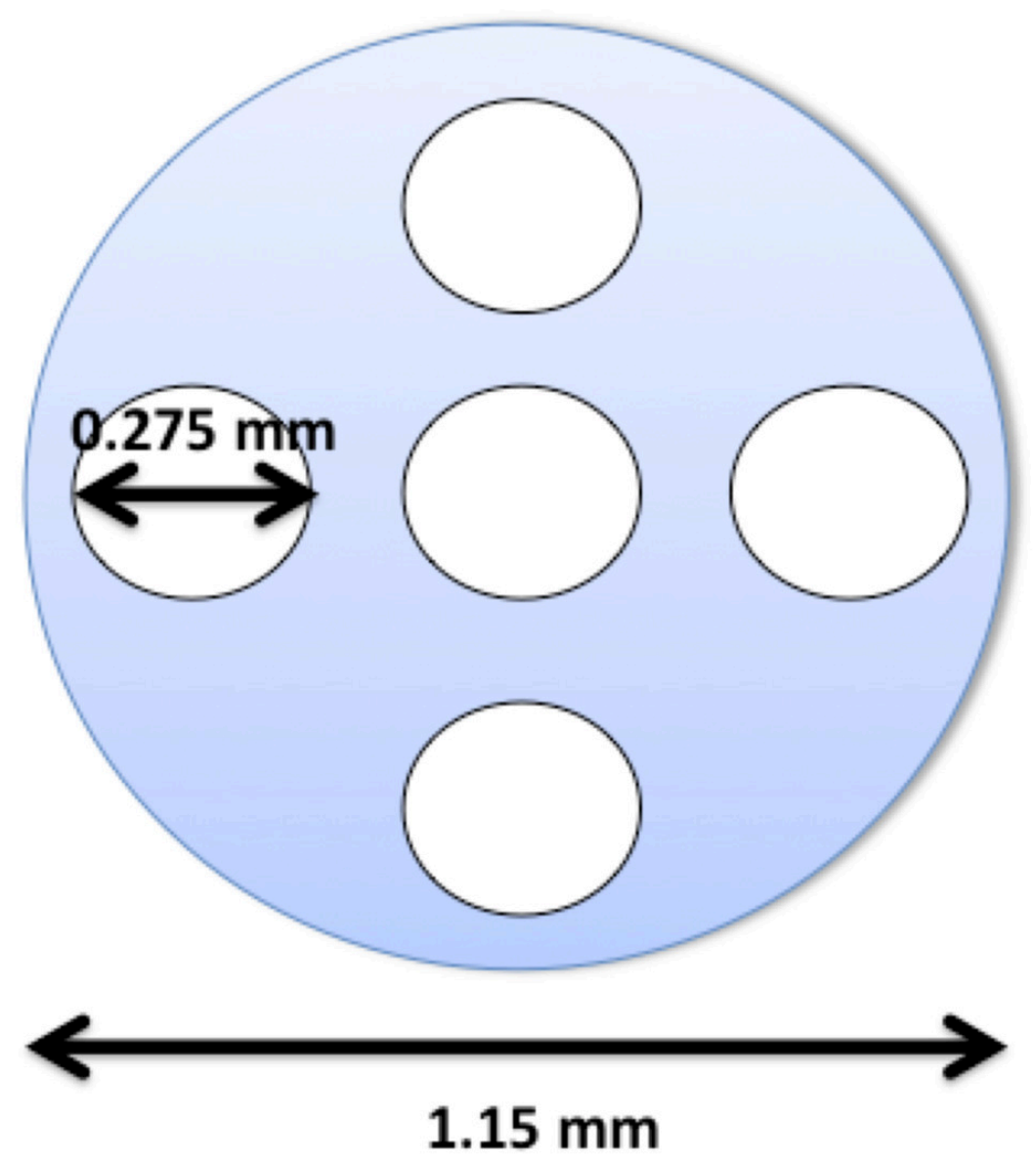

Figure 3.2: Multi-luminal catheter's cross sections with dimensions.

is influenced by both strain and temperature. Therefore, the thermal sensing catheter requires multiple FBG sensors within the catheter to compensate any discrepancies in the measurand due to any strain applied on the catheter. These reference FBG readings would then be subtracted from the measurement of the wavelength that reflects the temperature reading. Figure 3.3 is an example of the FBG sensor that was fabricated, which only consisted of a single FBG sensor. 


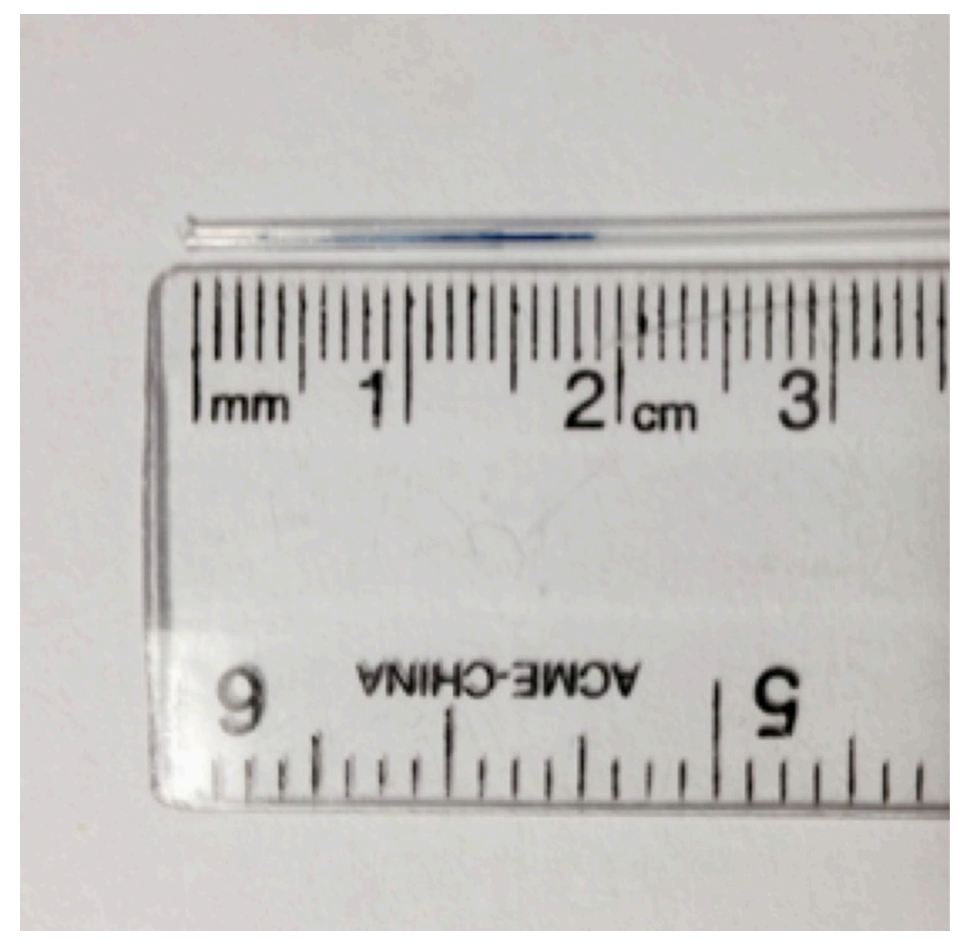

Figure 3.3: FBG thermal sensing catheter. This medical grade, multi-luminal catheter has the capability of housing five different functionalities. In this image, only one of the cavities is occupied with the FBG sensor (indicated by the dark blue region). The diameter of the catheter measures just under the $1.5 \mathrm{~mm}$ mark.

\subsection{Results and Discussion: FBG Calibration and Probe Fab- rication}

The FBG sensor calibration was determined based on five trials, which analyzed the sensor response between $25^{\circ} \mathrm{C}$ to $60^{\circ} \mathrm{C}$. The thermal sensors calibration curve was determined by obtaining the line of best fit from the average of the five trials, which produced the following linear relationship:

$$
\lambda_{b}=0.010245 t+1290.2
$$

where, $t$ is the temperature and the $\lambda_{b}$ is the shifted bragg wavelength. The temperature was the independent variable, and the resulting Bragg wavelength shift was dependent variable that shifted based on controlled temperature shifts. The calculated line of best fit has a regression value of 
0.9989, which shows a strong correlation between the data set and the linear fit. The rate of change of the wavelength with respect to temperature is $0.010245 \mathrm{~nm} /{ }^{\circ} \mathrm{C}$ for this particular FBG. The shift of the Bragg wavelength displaces based on the reference central Bragg wavelength, which was set at 1290.395 at room temperature and in a stress-free environment. Figure 3.4 shows the results of the calibration curve based on the five trials and its precise values are presented in Table 3.1. Based on the average results in Table 3.1, the maximum error observed is $2 \%$ at the temperature setting of $60^{\circ} \mathrm{C}$, which is considerably low. It is key to note that for every new FBG sensor that is integrated to this system, it will have to be calibrated, and similar to the results of this research, will have comparable linear trends with respect to incrementing temperature.

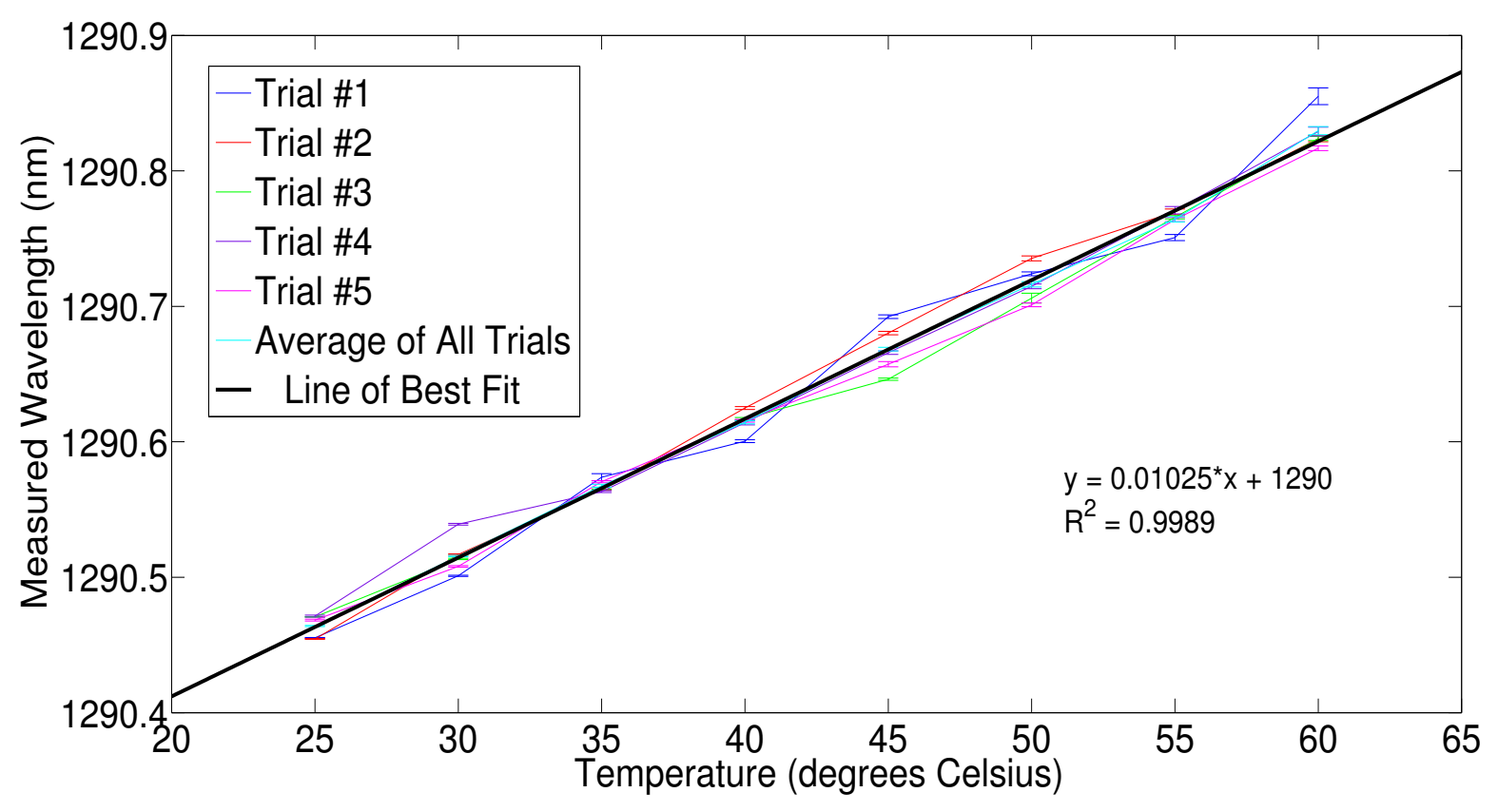

Figure 3.4: The calibration curve (black line) along with results of the five trials [32]. The quantities of the five trials at each temperature increment exhibit that the results were within close proximity. The calibration curve is represented by the following equation: $b=0.010245 t+1290.2$ (equation 3.1) 
Table 3.1: Thermal Look-up Table based on FBG reading [32].

\begin{tabular}{|c|c|c|c|c|c|}
\hline Temp./Trials & & $30^{\circ} \mathrm{C}$ & $40^{\circ} \mathrm{C}$ & $5^{\circ}{ }^{\circ} \mathrm{C}$ & $60^{\circ} \mathrm{C}$ \\
\hline 1 & $\begin{array}{c}\lambda \\
\text { Error }[\%]\end{array}$ & $\begin{array}{c}1290.501 \\
0.78\end{array}$ & $\begin{array}{c}1290.6005 \\
1.03\end{array}$ & $\begin{array}{c}1290.724 \\
0.85\end{array}$ & $\begin{array}{c}1290.8551 \\
2.7\end{array}$ \\
\hline 2 & $\begin{array}{c}\lambda \\
\text { Error }[\%]\end{array}$ & $\begin{array}{c}1290.5167 \\
0.77\end{array}$ & $\begin{array}{c}1290.6249 \\
0.92\end{array}$ & $\begin{array}{c}1290.7354 \\
1.1\end{array}$ & $\begin{array}{c}1290.8237 \\
1.0\end{array}$ \\
\hline 3 & $\begin{array}{c}\lambda \\
\text { Error }[\%]\end{array}$ & $\begin{array}{c}1290.5135 \\
0.75\end{array}$ & $\begin{array}{c}1290.6171 \\
0.81\end{array}$ & $\begin{array}{c}1290.7061 \\
2.3\end{array}$ & $\begin{array}{c}1290.8239 \\
0.71\end{array}$ \\
\hline 4 & $\begin{array}{c}\lambda \\
\text { Error }[\%]\end{array}$ & $\begin{array}{c}1290.5391 \\
0.86\end{array}$ & $\begin{array}{c}1290.6142 \\
1.6\end{array}$ & $\begin{array}{c}1290.7148 \\
1.1\end{array}$ & $\begin{array}{c}1290.8291 \\
1.5\end{array}$ \\
\hline 5 & $\begin{array}{c}\lambda \\
\text { Error }[\%]\end{array}$ & $\begin{array}{c}1290.5081 \\
0.95\end{array}$ & $\begin{array}{c}1290.6156 \\
0.93\end{array}$ & $\begin{array}{c}1290.7011 \\
0.90\end{array}$ & $\begin{array}{c}1290.8167 \\
4.0\end{array}$ \\
\hline Average & $\begin{array}{c}\qquad \\
\text { Error }[\%]\end{array}$ & $\begin{array}{c}1290.5157 \\
0.82\end{array}$ & $\begin{array}{c}1290.6144 \\
1.1\end{array}$ & $\begin{array}{c}1290.7163 \\
1.3\end{array}$ & $\begin{array}{c}1290.8297 \\
2.0\end{array}$ \\
\hline
\end{tabular}

To verify whether the thermal FBG system was precise, a blind test was conducted to assess its performance. The blind test consisted of three temperature trials where the temperature of the digital hotplate was randomly set and after a certain point in time, the FBG measurement from the spectrometer was processed using equation 3.1. The outcomes of the FBG system were then compared to the readings obtained from the thermocouple, which are shown in table 3.2. The percentage of error was highest in the first trial corresponding to $3.6 \%$, the second at $1.7 \%$ and the last at $1.1 \%$. The sources of error could be due to the limitations in control with the phantom 
temperature. Due to potential variations in room temperature and the lack of feedback from the hotplate, it was difficult to maintain a constant temperature. However, the error is minute and can be validated to provide relative local temperature of any sample.

Table 3.2: Blind Test Results [32]; randomized hot plate temperature settings and resulting FBG measurements are shown above. Using equation 3.1 the temperature was determined by observing the Bragg wavelength readings from the spectrometer.

\begin{tabular}{|c||c|c|c|c|}
\hline Blind Test Trials & Thermocouple $\left[{ }^{\circ} \mathbf{C}\right]$ & $\lambda[\mathbf{n m}]$ & Calibrated FBG $\left[{ }^{\circ} \mathbf{C}\right]$ & Error [\%] \\
\hline \hline $\mathbf{1}$ & 36 & 1290.55 & 34.7 & 3.6 \\
\hline $\mathbf{2}$ & 29 & 1290.50 & 29.5 & 1.7 \\
\hline $\mathbf{3}$ & 27 & 1290.48 & 27.3 & 1.1 \\
\hline
\end{tabular}

After the calibration process and the blind test, an initial attempt was made to fabricate a thermal sensing catheter using multi-luminal tubing. The multiple holes are convenient for housing multiple components in one entity (such as: the FBG sensor and OCT imaging fiber), which is an essential feature for clinical practice. Figure 3.3 is an image of the catheter that within the FBG sensor, which is highlighted as the dark region. As can be seen in the image, the catheter is dimensionally small and complies with the design requirements to a diameter either equal to or smaller than $1.5 \mathrm{~mm}$ threshold.

\subsection{Chapter 3 Summary}

This chapter introduced the application of using optical sensors, FBG, in monitoring temperature changes in a controlled environment. The thermal response calibration curve was developed with controlled temperature settings, by which the results indicated a linear trend with a regression value of 0.9989 . This preliminary work could lead to the development of a thermal sensitive probe, where 
the FBG sensor could provide a reference measurement for clinicians while performing thermal ablative procedure. This will however require a much further analysis and greater collaboration amongst engineers and clinicians. 


\section{Chapter 4}

\section{OCT Envelope Statistics}

7 HE following chapter provides a description of the thermal-OCT imaging setup and ana-

L lyzes the various PDFs that were mentioned in Chapter 2. The best fit to the backscattered envelope of the OCT images was determined based on the correlation factor from the KS test. Moreover, the results of the best fit PDF parameters were observed with respect to changing temperature on the tissue-mimicking phantom.

\subsection{Experimental Procedure}

\subsubsection{Hot Plate Calibration Process - Thermo Scientific ELED HP130910- 33Q Cimarec}

Prior to beginning any thermal-control research with the tissue-mimicking phantom (microsphere suspension), a new digital hotplate was purchased from Thermo Scientific for the FBG-OCT system. The variation of thermal feedback between the digital hotplate and thermocouple required another calibration curve to compensate for the offset. Similar to the calibration process of the FBG system, five trials were conducted by measuring the temperature of the sample (via thermocouple) by setting the hotplate thermal settings between $25^{\circ} \mathrm{C}-80^{\circ} \mathrm{C}$, at $5^{\circ} \mathrm{C}$ increments.

\subsubsection{OCT-Thermal Imaging Setup with Tissue-Mimicking Phantoms}

The thermally controlled study on the phantoms was imaged using the Biophotonics and Bioengineering Laboratory's (BBL) in-house polygon swept-source OCT system, located at Ryerson 
University. As previously mentioned, the OCT system works on the basis of the interferometer, and the resulting fringe signal acquired from the constructive and destructive behavior of the light is converted into a grey scale image using the Thorlab Software via MATLAB. The in-house OCT system uses a Gaussian profile light source with a central wavelength of $1310 \mathrm{~nm}$ and corresponding axial resolution of $6.9 \mu \mathrm{m}$ in the air medium, but this axial resolution is subject to change with respect to the refractive index of the sample being imaged. The transverse resolution of the system is approximately $20 \mu \mathrm{m}$. The system has an A-scan rate of $72 \mathrm{kHz}$ and penetration depth of approximately $1.4 \mathrm{~mm}$. In the top left corner Figure 4.1 of the optical schematic of the polygon swept source; in addition, the image also exhibits the imaging and thermal setup of this study. Table 4.1 outlines the description of all components that were applied within the optical setup and are described in the Figure 4.1 decription. The temperature of the phantom was controlled using a digital hotplate made by Thermo Scientific (ELED HP130910-33Q Cimarec) and apart from the feedback from the digital hotplate, the temperature was also monitored using a thermocouple (via multimeter).

The first phantom to be fabricated for the thermal imaging process was a titanium dioxide phantom, which mimicked the optical properties of biological tissue. The refractive index of the phantom was achieved by mixing $0.665 \mathrm{~g}$ of titanium dioxide $\left(\mathrm{T}_{i} \mathrm{O}_{2}\right.$ - Titanium (IV) oxide, anatase) that has a particle size smaller than $25 \mathrm{~nm}$ with $475 \mathrm{~mL}$ of Penecros Versagel (hydrocarbon material). Due to cells being made up of various components, the refractive index can vary according to the microstructures. The refractive index value that is commonly acknowledged as the norm falls within the close range of $1.4-1.5$ [50]. The concentration of titanium dioxide $(0.665 \mathrm{~g})$ with respect to Versagel $(475 \mathrm{~mL})$ resulted in a refractive index of approximately 1.4. OCT images of the titanium dioxide phantom were taken of the phantom at varying temperature settings and an example is given in Figure 4.2 a). The red box in the top image is the ROI to study OCT envelope statistics; the ROI observes a region that consists of $700 \mathrm{~A}$-scans and a depth of $\sim 0.4 \mathrm{~mm}$, which analyzes 42000 pixels when mapping the histogram. The PDFs that were discussed in section 2.2 were fit with the histograms of the empirical data acquired at temperatures at approximately $25^{\circ} \mathrm{C}$, 


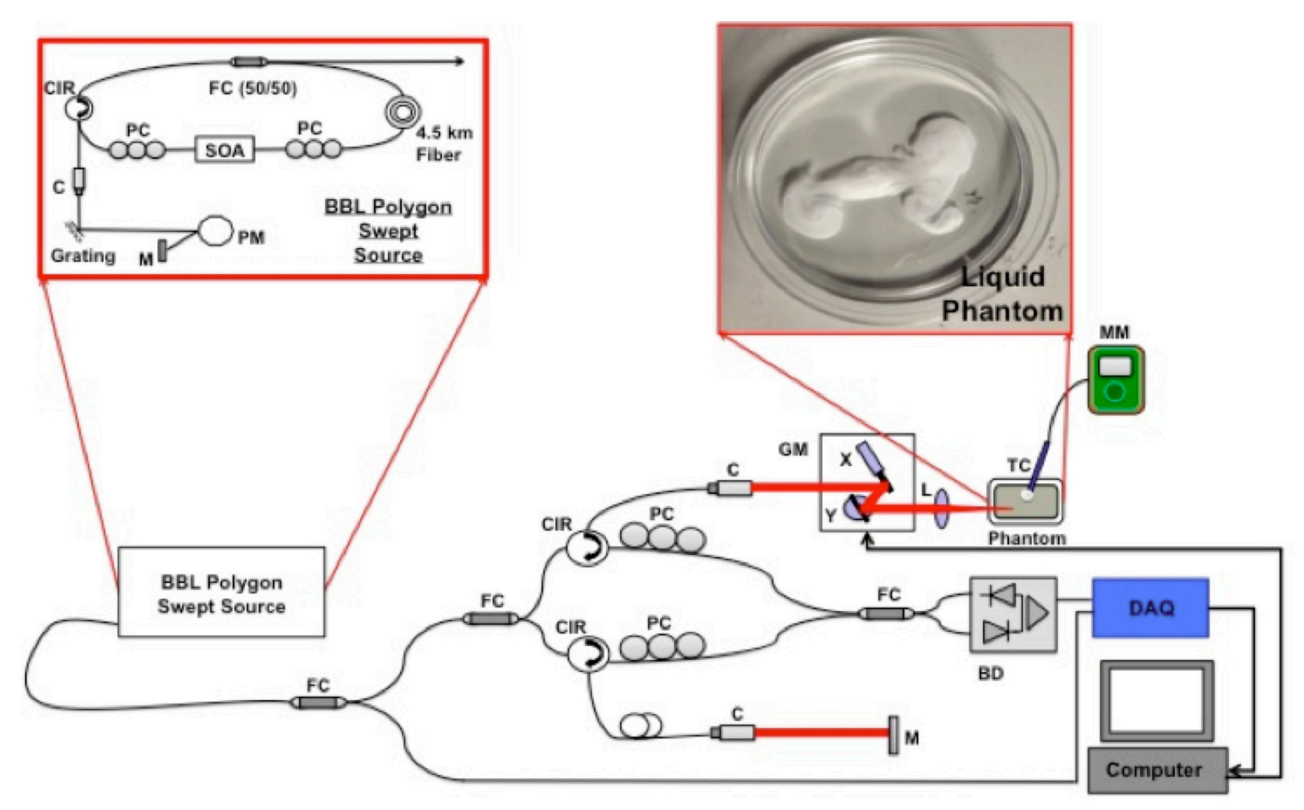

Figure 4.1: The Schematic of the temperature measurement and OCT systems [49]; Circulator (CIR); Fiber Coupler (FC); Collimator (C); Balanced Detector (BD); Mirror (M); Polarization Controller (PC); Thermocouple (TC); Galvo Mirrors (GM); Lens (L); Multi-meter (MM); Polygon Mirror (PM); Semiconductor Optical Amplifier (SOA).

$30^{\circ} \mathrm{C}, 35^{\circ} \mathrm{C}, 40^{\circ} \mathrm{C}, 45^{\circ} \mathrm{C}, 50^{\circ} \mathrm{C}, 55^{\circ} \mathrm{C}$ and $60^{\circ} \mathrm{C}$. The parameters of the most apt PDF were analyzed with respect to the incremented temperature settings to look for a correlation between temperature and the PDF parameters.

The phantom was a colloidal suspension fluid that is composed of polystyrene microspheres (Polysciences, Warrington, PA) and glycerin USP (topical skin emollient), which is widely used for pharmaceutical and cosmetic reasons. Glycerin is a sugar alcohol based compound that is transparent and colorless, and has a refractive index of approximately 1.46 [51]. In the phantom, polystyrene microbeads with a mean diameter of $0.75 \mu \mathrm{m}$ with deviation of $0.025 \mu \mathrm{m}$ were used. The polystyrene beads refractive index is $\sim 1.59$ [52], which resulted in the phantom having an overall refractive index of approximately 1.47. The overall refractive index of the phantom was calculated based on the percentage concentration with reference to the solute and solvents mass. For every gram of glycerin USP, $0.056 \mathrm{~g}$ of polystyrene beads were included in the monodisperse 
Table 4.1: List of all the Optical and Electrical Components in the Thermal OCT Sensing System

\begin{tabular}{|c|c|}
\hline Optical Components & Description \\
\hline Circulator & $\begin{array}{l}\text { Three to four-port device, that takes a light wave and } \\
\text { transmits to the next port in rotation }\end{array}$ \\
\hline Fiber Coupler & $\begin{array}{l}\text { Device that houses one or more input optical fibers } \\
\text { and has one or several outputs (power is distributed) }\end{array}$ \\
\hline Collimator & Focuses the light waves \\
\hline Balanced Detector & $\begin{array}{l}\text { Measure of light intensity } \\
\text { - consists of two photodiode and amplifier, to elimi- } \\
\text { nate common mode noise }\end{array}$ \\
\hline Mirror & $\begin{array}{l}\text { A surface that reflects light and conserves the input } \\
\text { effectively with little loss }\end{array}$ \\
\hline Polarization Controller & $\begin{array}{l}\text { Modify polarization state of light (three waveplates) } \\
\text { - linear, circular and elliptical }\end{array}$ \\
\hline Thermocouple & $\begin{array}{l}\text { Device that responds to change in temperature by pro- } \\
\text { ducing a current }\end{array}$ \\
\hline Galvo Mirrors & Reflective surfaces that detect position \\
\hline Lens & $\begin{array}{l}\text { An optical medium that transmits or refracts light } \\
\text { - light can either converge or diverge }\end{array}$ \\
\hline Multi-meter & $\begin{array}{l}\text { An electronic measuring device that is capable of } \\
\text { measuring various metrics }\end{array}$ \\
\hline Polygon Mirror & $\begin{array}{l}\text { Mirror with multiple facets; commonly used for scan- } \\
\text { ning purposes }\end{array}$ \\
\hline Semiconductor Optical Amplifier & $\begin{array}{l}\text { An active gain medium that amplifies the signal with- } \\
\text { out converting it into an electrical signal }\end{array}$ \\
\hline
\end{tabular}

phantom $(5.6 \%$ w/w). Figure 4.3 is an OCT image of the microsphere suspension phantom. Figure 4.1 displays an image of the physical phantom prior to the solution being mixed until it was homogeneous. The phantom was thoroughly mixed and was imaged after a span of 24 hours. The region of consisted was chosen $30 \mu \mathrm{m}$ below the surface of the phantom to avoid pixel intensities from the surface reflection. The region of interest had a transverse distance of $293 \mu \mathrm{m}$ and a depth of $175 \mu \mathrm{m}$, which contained 1500 pixels. 


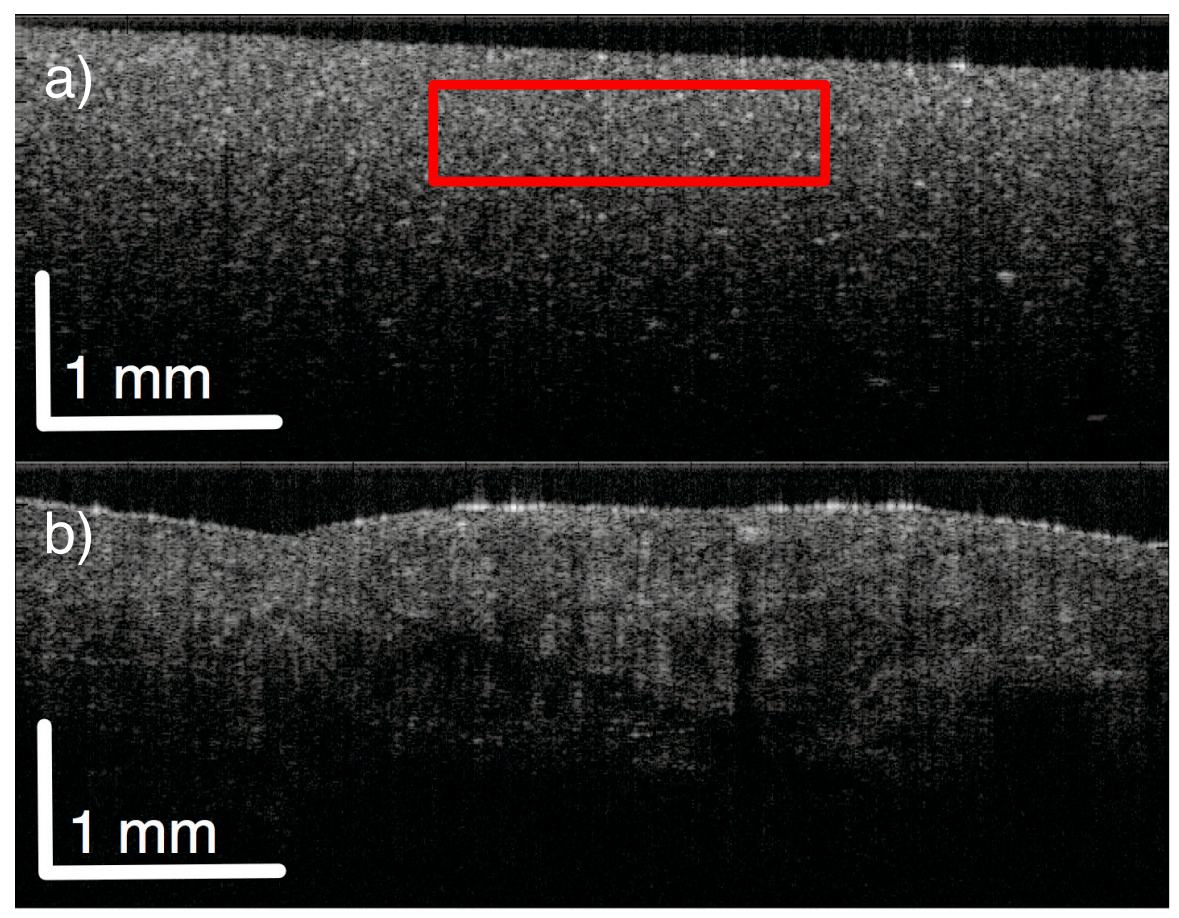

Figure 4.2: OCT image samples of: a) a titanium dioxide phantom that mimicks the refractive index of biological tissue and b) chicken tissue.

For this particular research setup, the OCT system acquired $3 \mathrm{~mm} \times 3 \mathrm{~mm}$ images (corresponding to the depth and transverse distance), as seen in Figure 4.3. The phantom was imaged at the following temperature settings: $22^{\circ} \mathrm{C}, 27^{\circ} \mathrm{C}, 31^{\circ} \mathrm{C}, 35^{\circ} \mathrm{C}, 40^{\circ} \mathrm{C}, 42^{\circ} \mathrm{C}, 47^{\circ} \mathrm{C}, 52^{\circ} \mathrm{C}$ and $57^{\circ} \mathrm{C}$.

\subsection{Results and Discussion}

\subsubsection{Hot Plate Calibration Curve}

The average of the five trials was set as the calibration fit, along with the standard deviation of the results at each hot plate temperature setting. Figure 4.4 provides a visual representation of the linear offset between the two thermal measurements. The linear trend is denoted by the following equation: 


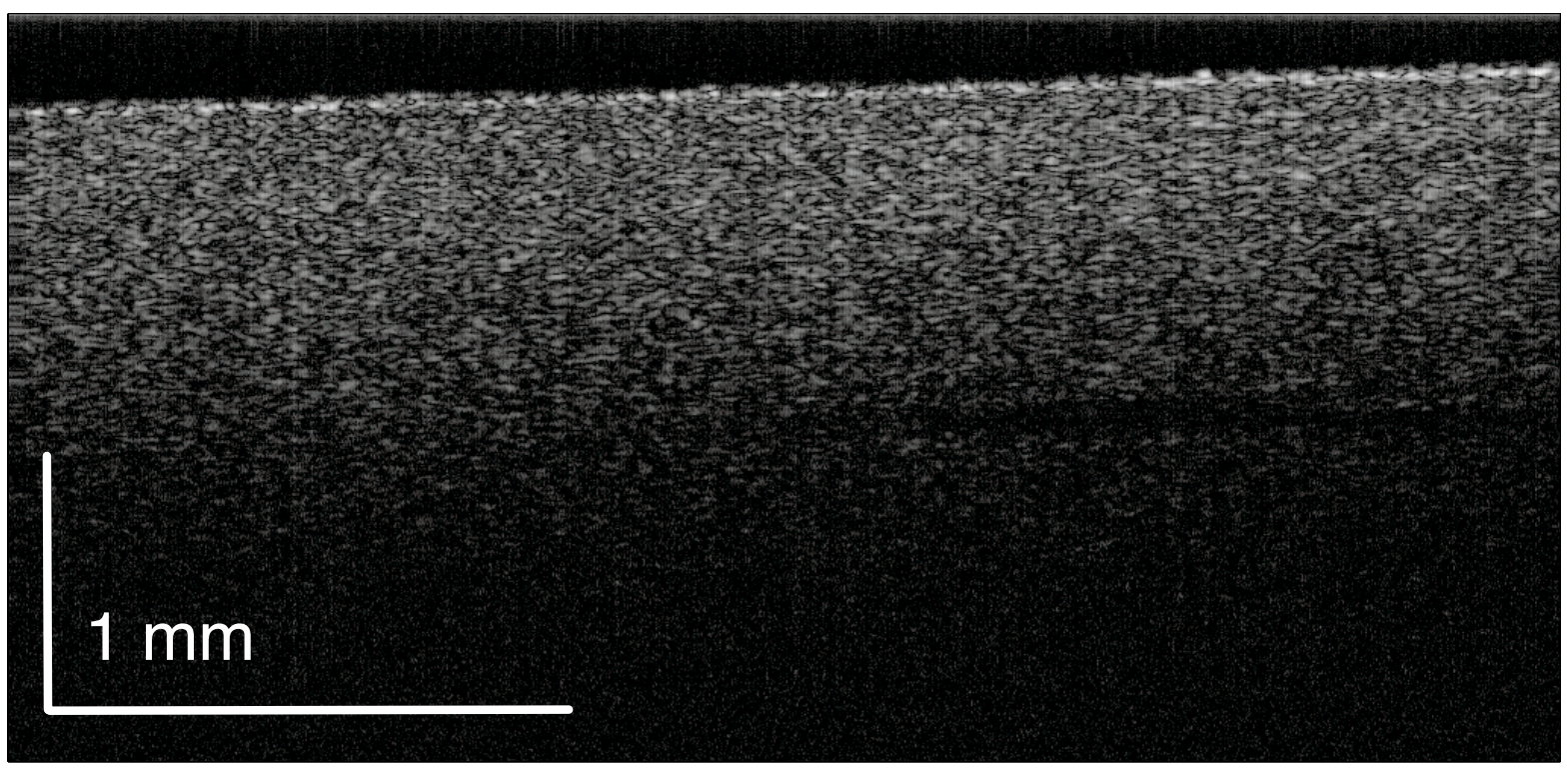

Figure 4.3: An example of an OCT image of the tissue-mimicking phantom with $3 \mathrm{~mm} \times 3 \mathrm{~mm}$ dimensions. The white granules in the image are referred to as speckle.

$$
T_{s}=0.010245 \times T_{h}+1290.2
$$

where, $T_{s}$ is the sample temperature and $T_{h}$ is the hot plate temperature setting. The slope of the linear trend is close to a value of one, which conveys that the error in hot plate temperature feedback is constant amongst all temperatures. The calibration curve had a linear regression value of 0.9951 that represented the experimental data accurately.

The calibration curve in Figure 4.4 of the measured sample temperature (via thermocouple) and the hotplate indicate there were discrepancies in the actual hot plates temperature and in the value conveyed through the feedback. Prior to starting any experimental work, a calibration curve was required to compensate for the offset and to accurately control the temperature settings on the phantom. 


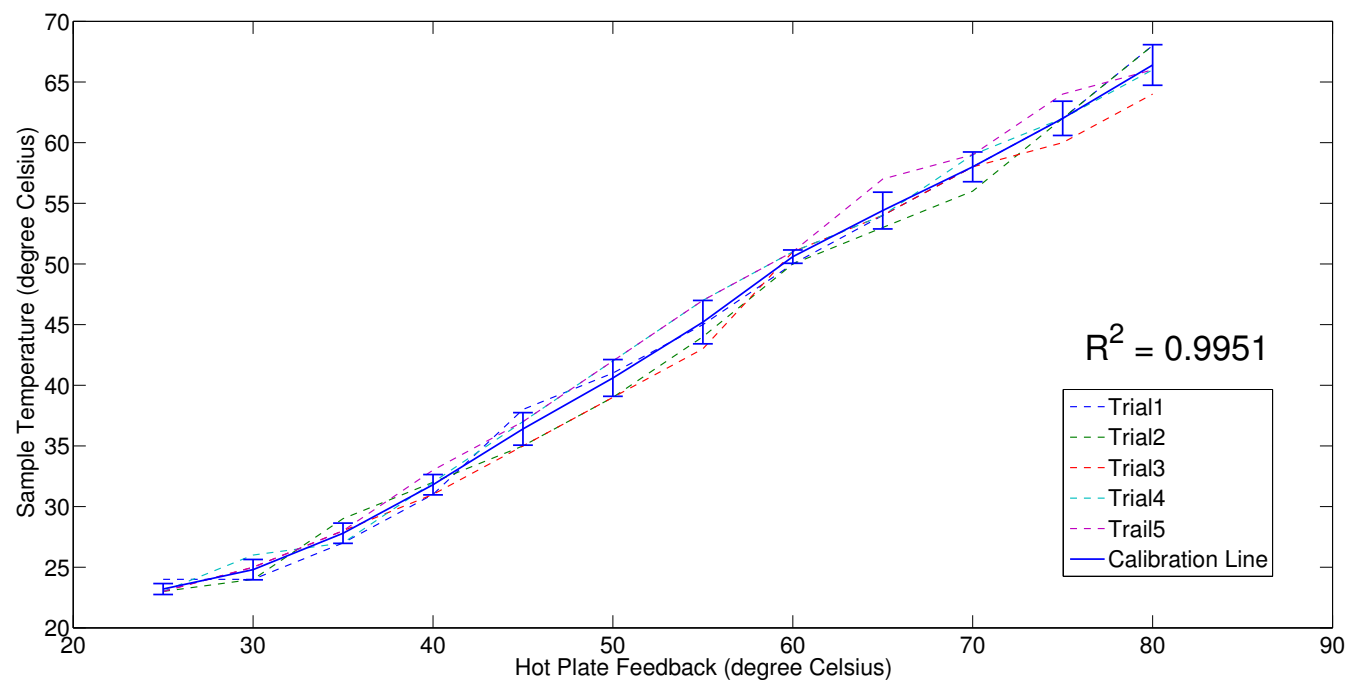

Figure 4.4: The calibration curve of the digital hot plate [49]. The calibration curve was configured based on the mean of all five trials. At each hot plate thermal setting, the standard deviation is represented with the error bars to indicate the variance in the thermocouple readings.

\subsubsection{OCT Speckle and Envelope Statistics}

OCT envelope statistics provides a metric to analyze structural changes in the scatterer of sample, due to corresponding variations in speckle. The envelope of the histogram was fit to four PDFs, and the goodness of fit is determined based on the correlation factor of the KS test. The correlation factor below enables one to either accept or reject whether a particular PDF is apt to represent the intensity distribution of the empirical data.

The initial phantom that was imaged using the FBG-OCT system was a titanium dioxide phantom and the generalized gamma fit provided the most accurate fit to the data set, but this agreement was further strengthened when the correlation factor of the generalized gamma function was the smallest grade that was under the 0.05 . Figure 4.5 provides the different PDF fits for the OCT image of the titanium dioxide phantom, and the corresponding correlation factors are given in Table 4.2. The primary research conducted with the titanium dioxide phantom established that the generalized gamma function was the best representative for the envelope statistics of the OCT images of tissue-mimicking phantoms. 
Table 4.2: The corresponding correlation factor for the four different PDFs of an OCT image, of the titanium dioxide phantom.

\begin{tabular}{|l||l|}
\hline Probability Distribution Functions & Correlation Factor $(\mathbf{p}<\mathbf{0 . 0 5})$ \\
\hline \hline Rayleigh & 0.0847 \\
\hline Rician & 0.085 \\
\hline Generalized Gamma & 0.0158 \\
\hline Normal & 0.1176 \\
\hline
\end{tabular}

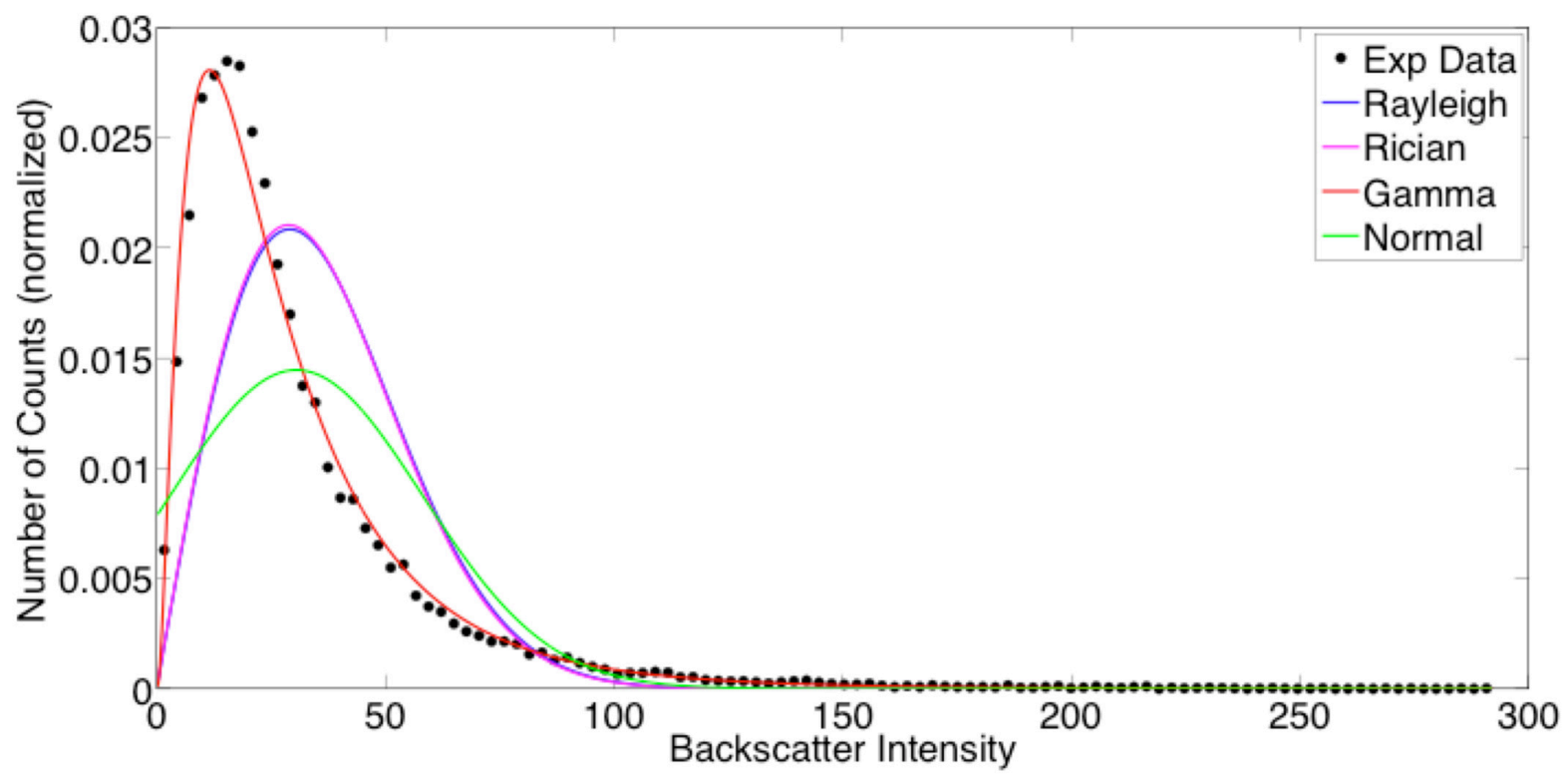

Figure 4.5: The Rayleigh, Rician, Generalized Gamma and Normal PDF fits of the envelope statistics of an OCT image of the titanium dioxide tissue mimicking phantom.

Even though the titanium dioxide provided essential information with respect to envelope statistics analysis, the phantom was not ideal for thermal experiments. The Versagel base used in the phantom did not convey any viable information with modulation to temperature. The general- 
ized gamma parameters (two shape parameters $c$ and $v$ and the scale parameter $a$ ) were analyzed with respect to changing temperature and the data was not statistically significant as there were no apparent trends. Figure 4.6 shows the how the generalized gamma parameters changed with respect to temperature, which does not convey any obvious trends. However, it was apparent that changes in temperature were influencing the optical properties and structural integrity of the phantom, as there were fluctuations in the parameters. The most evident alteration can be observed post $40^{\circ} \mathrm{C}$, where there is a drastic increase in quantity for the shape parameter $c$ (Figure 4.10a) and scale $a$ (Figure 4.10d), and a decrease in shape parameter $v$ (Figure 4.10b). These extreme changes may be a result of the Versagel starting to melt as it reaches closer to its melting point.

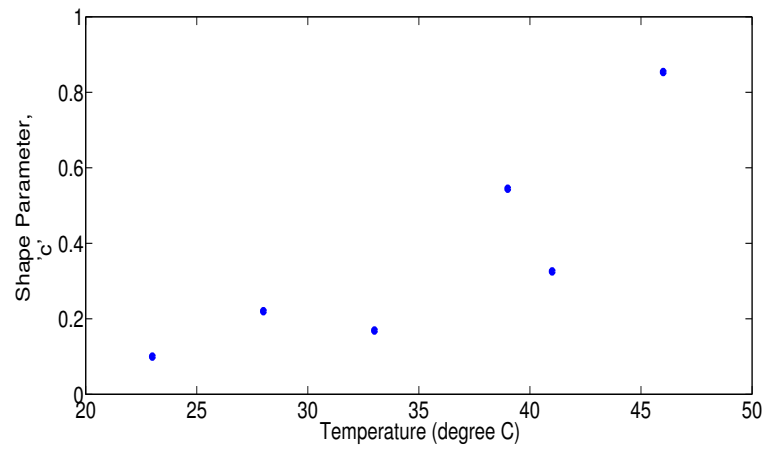

(a) The shape parameter $c$

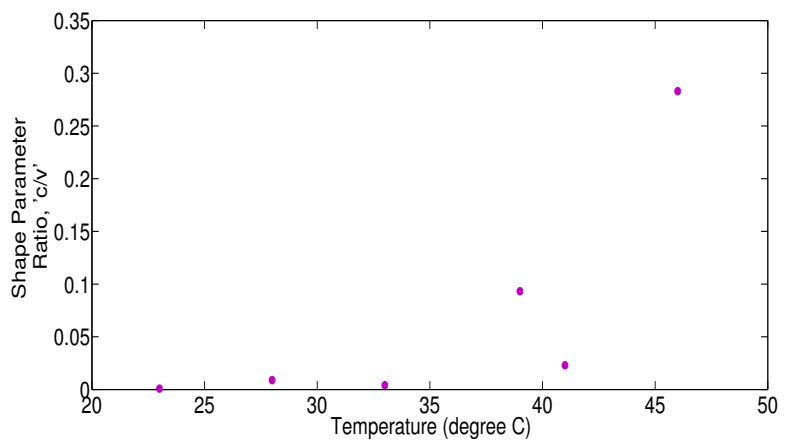

(c) The shape parameter ratio $c / v$

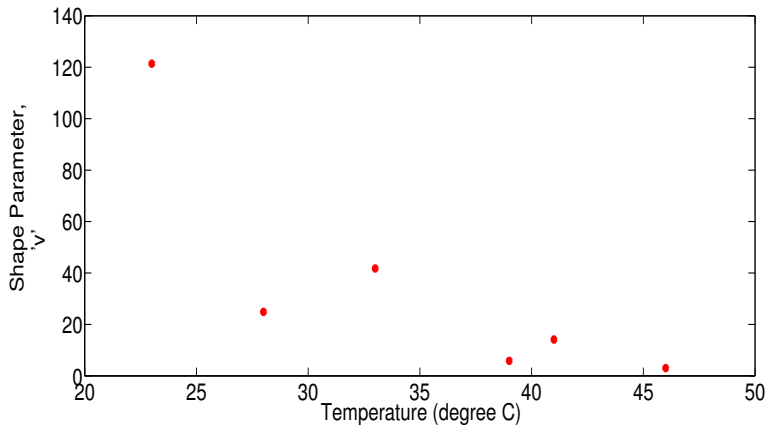

(b) The shape parameter $v$

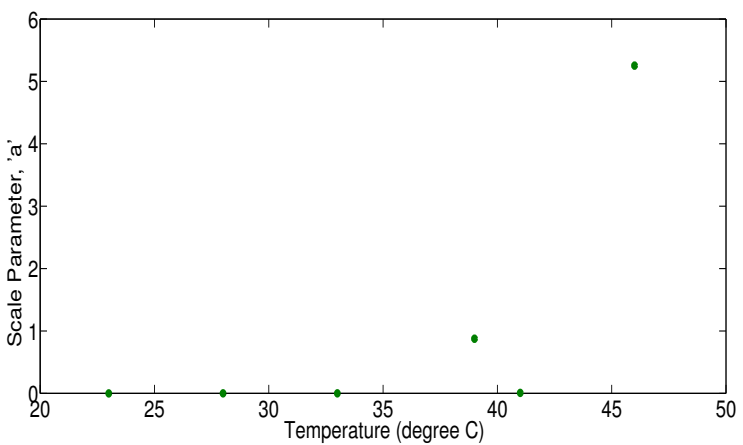

(d) The scale parameter ratio $a$

Figure 4.6: The results of the three generalized gamma parameters for the titanium dioxide phantom with respect to modulating temperature [32]. 
The next phase required the fabrication of another phantom that was more apt to analyzing the changes in temperature, but with optical properties of similar nature. Based on prior research on envelope statistics, a commonly used phantom for initial study that made comparison to analyze the US backscatter spectrum between cell pellets and phantoms, had utilized a microsphere suspension phantom which exhibited the potential to determine scatterer size [52]. An OCT image of the microsphere suspension phantom was acquired and the PDF distribution fits were examined accordingly. Due to using a phantom of similar optical properties as the titanium dioxide, but with different materials, it was imperative to assess the PDFs fits once again prior to running any thermal imaging trials. The results of this analysis are provided in Figure 4.7, which outlines the ROI of an OCT image of the tissue-mimicking phantom and the corresponding PDF fits to the empirical data. Moreover, Table 4.3 provides the correlation factor for each PDF, which indicates that the generalized gamma distribution was the best fit.

Table 4.3: The corresponding correlation factor for the four different PDFs of an OCT image of the microsphere suspension phantom.

\begin{tabular}{|l||l|}
\hline Probability Distribution Functions & Correlation Factor $(\mathbf{p}<\mathbf{0 . 0 5})$ \\
\hline \hline Rayleigh & 0.3090 \\
\hline Rician & 0.2956 \\
\hline Generalized Gamma & 0.0236 \\
\hline Normal & 0.2712 \\
\hline
\end{tabular}

Figure 4.8 exhibits the generalized gamma fit along with the experimental data, which was established as the PDF whose parameters would be closely studied with respect to the quantifying local temperature changes in tissue mimicking fluid phantom using envelope statistics. OCT images of the microsphere phantom were acquired at varying temperatures that were modulated by the hot plate, and the generalized gamma function was surveyed at each thermal setting. The 


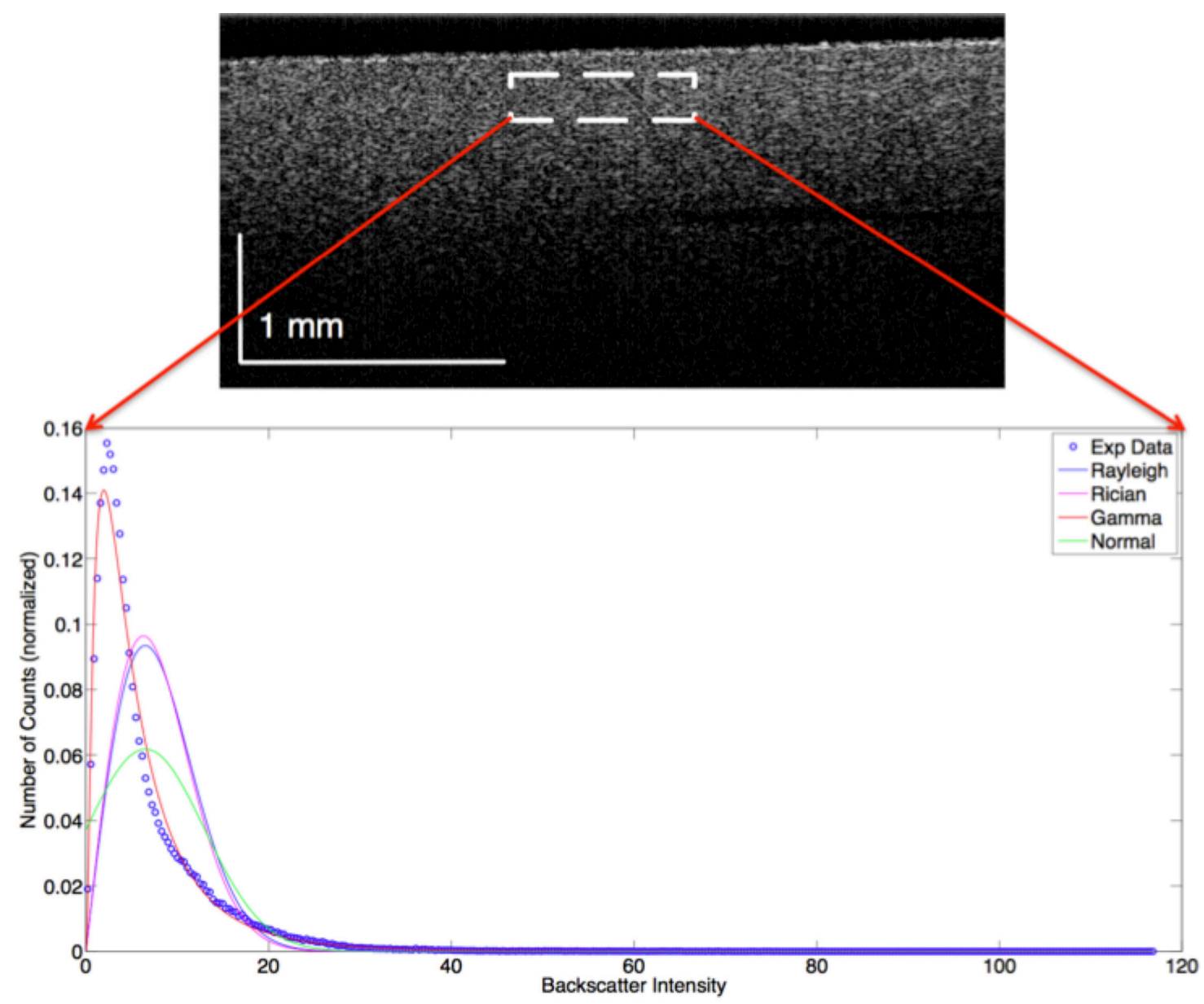

Figure 4.7: The Rayleigh, Rician, Generalized Gamma and Normal PDF fits of the envelope statistics for the outline ROI of an OCT image of the tissue mimicking phantom. The blue dotted markers represents the empirical data.

results of the changes in the generalized gamma function, with respect to changing temperatures, are depicted in Figure 4.9.

Similar to the analysis of the titanium dioxide phantom, the parameters of the generalized gamma functions were also analyzed, which are shown in Figure 4.10. The actual values of the parameters are given in Table 4.4. 


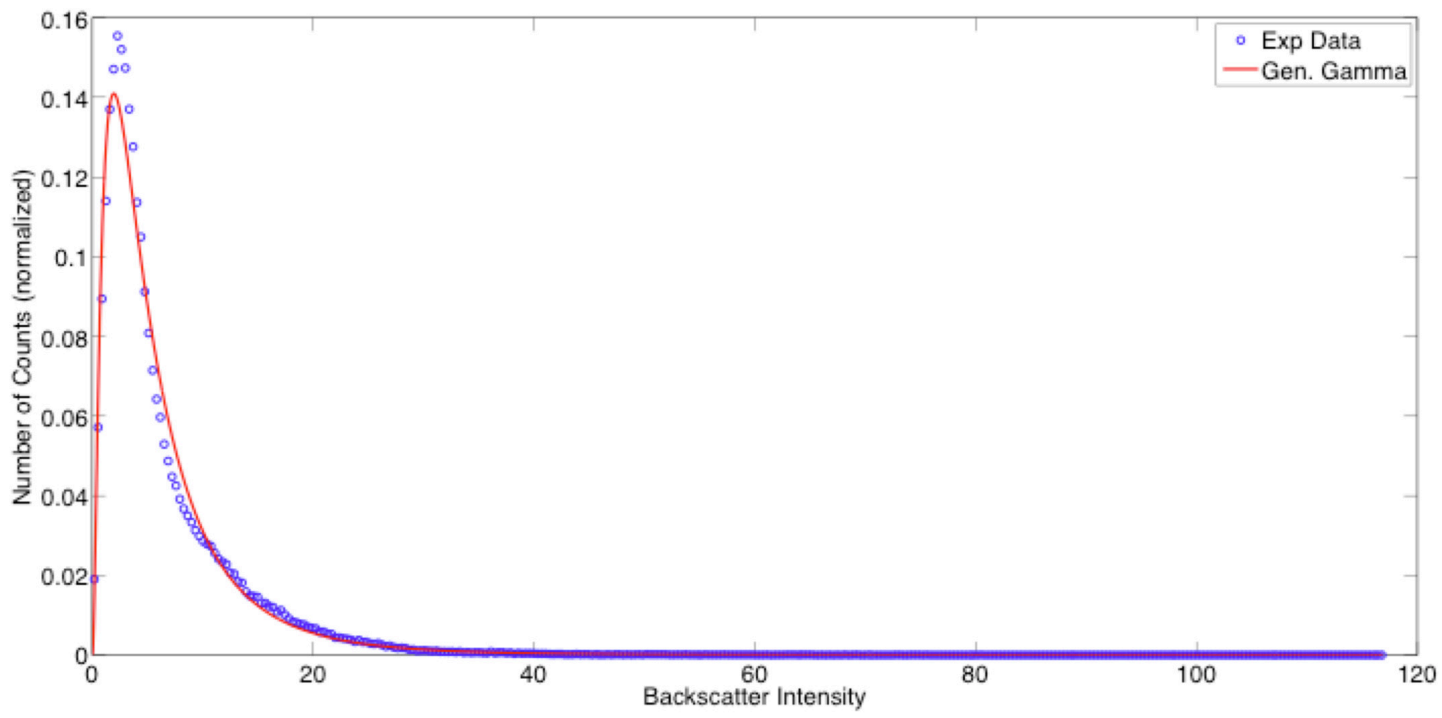

Figure 4.8: A closer look at the Generalized Gamma fit to the experimental data, which was determined to by using the KS test.

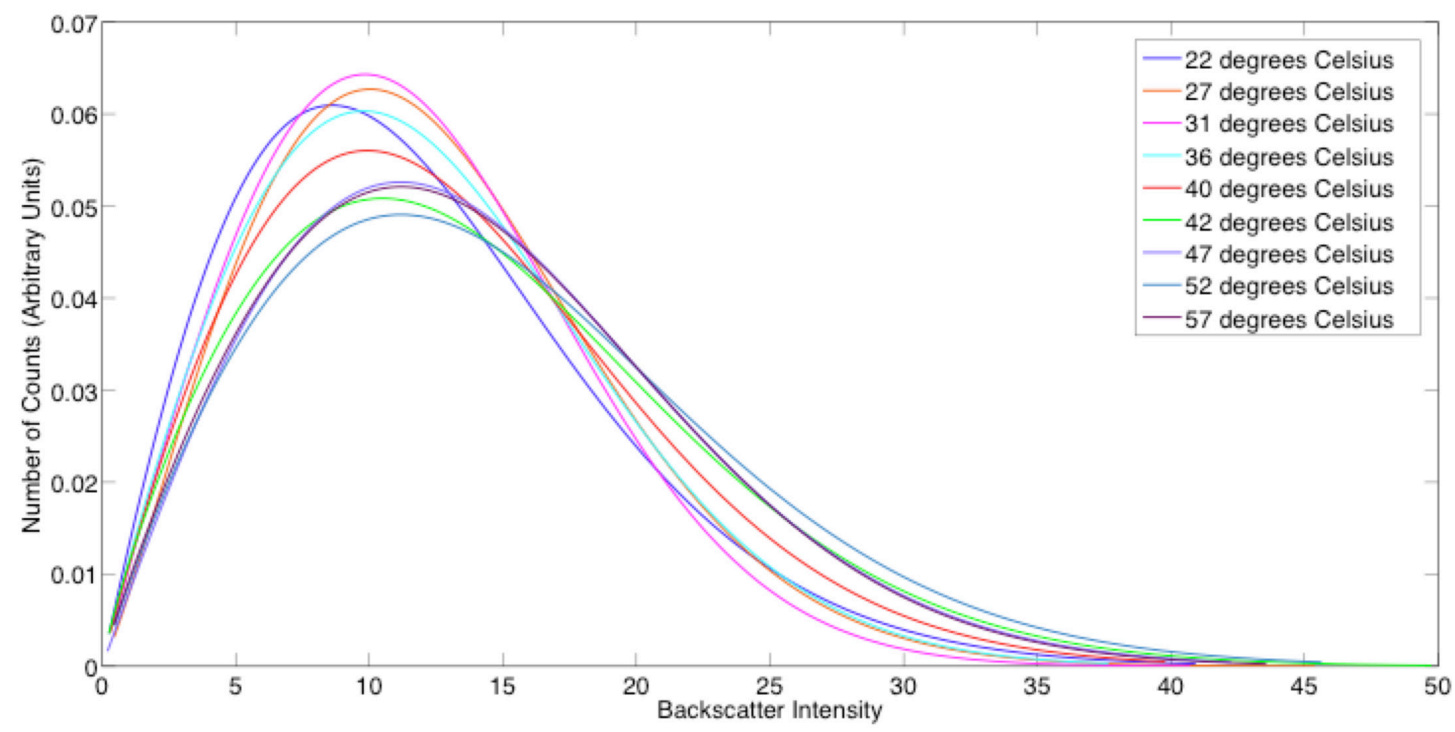

Figure 4.9: The Generalized Gamma distribution fits are shown above with respect to nine different temperature settings [49]. The changes with each peaks and position of the generalized gamma fits demonstrate that thermal changes influence the parameters that dictate the overall shape of the function. 
Table 4.4: The results of the two shape parameters $c$ and $v$, and the scale parameter $a$ with respect to changing temperature [49].

\begin{tabular}{|l||l|l|l|l|}
\hline $\begin{array}{l}\text { Sample Temper- } \\
\text { ature }\left[{ }^{\circ} \mathbf{C}\right]\end{array}$ & $\begin{array}{l}\text { Shape Parame- } \\
\text { ter, } c\end{array}$ & $\begin{array}{l}\text { Shape Parame- } \\
\text { ter, } v\end{array}$ & $\begin{array}{l}\text { Shape Parame- } \\
\text { ter Ratio, } c / v\end{array}$ & $\begin{array}{l}\text { Scale Parameter, } \\
a\end{array}$ \\
\hline $\mathbf{2 0}$ & 1.60308628 & 1.24570675 & 1.286888972 & 11.58558244 \\
\hline $\mathbf{2 4}$ & 1.761330096 & 1.199519305 & 1.468363276 & 12.03425875 \\
\hline $\mathbf{2 7}$ & 1.902795945 & 1.136540708 & 1.674199553 & 13.00433546 \\
\hline $\mathbf{3 1}$ & 2.165826656 & 0.917682546 & 2.360104444 & 14.14874521 \\
\hline $\mathbf{3 6}$ & 2.012804072 & 0.95966081 & 2.097411972 & 14.34817573 \\
\hline $\mathbf{4 0}$ & 1.83972433 & 1.048325298 & 1.754917423 & 14.40837338 \\
\hline $\mathbf{4 7}$ & 1.902348895 & 1.051711803 & 1.808811967 & 15.67281751 \\
\hline $\mathbf{5 2}$ & 1.812009994 & 1.059014799 & 1.711033685 & 16.26308481 \\
\hline $\mathbf{5 7}$ & 2.022470418 & 0.939209376 & 2.153375455 & 16.70370514 \\
\hline
\end{tabular}




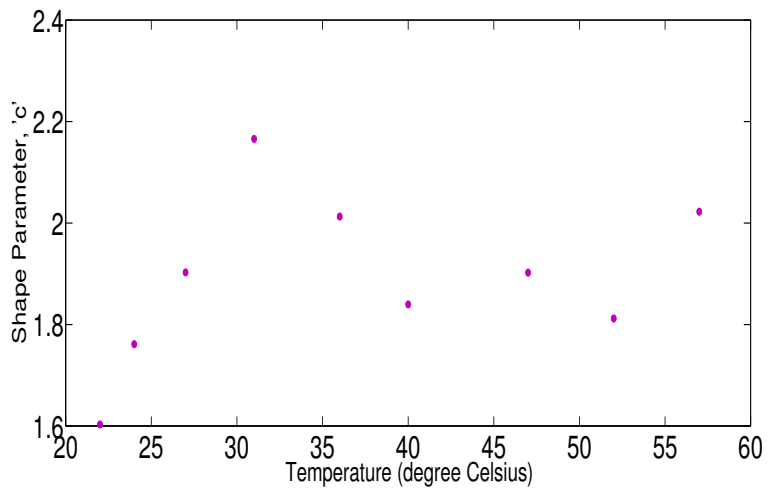

(a) The shape parameter $c$

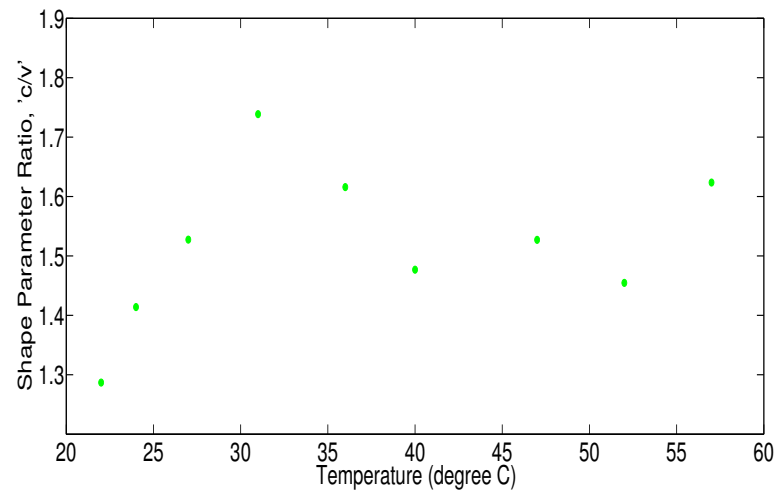

(c) The shape parameter ratio $c / v$

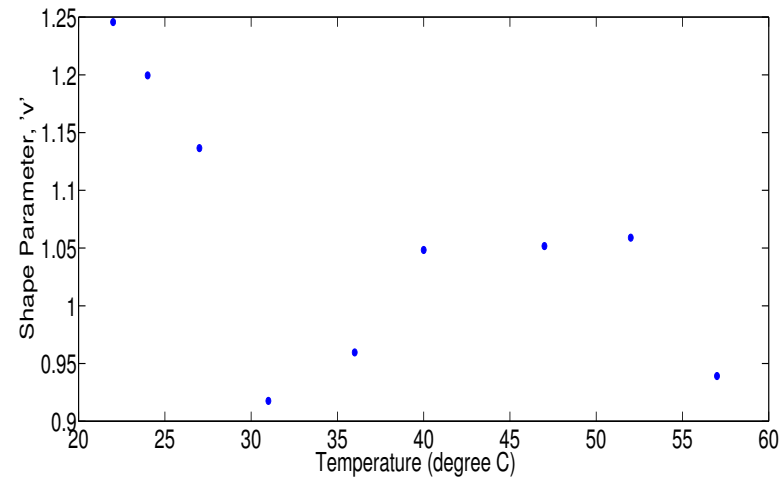

(b) The shape parameter $v$

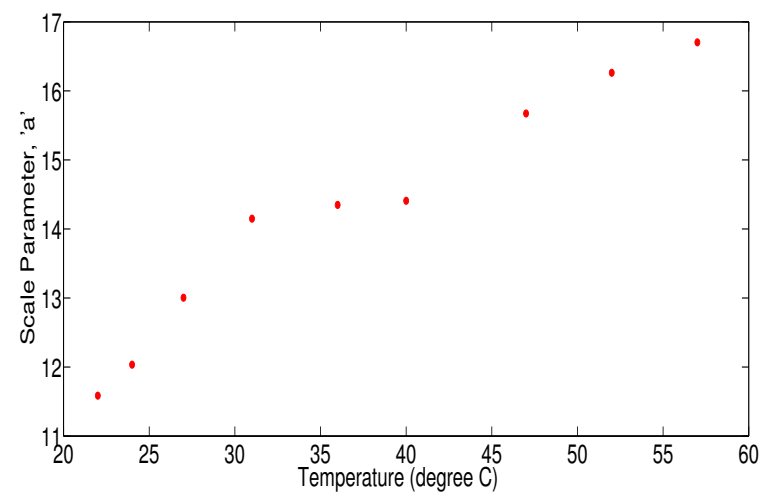

(d) The scale parameter ratio $a$

Figure 4.10: The three generalized gamma parameters observed within the range of $22^{\circ} \mathrm{C}-57^{\circ} \mathrm{C}$ range [49]. 
The shape parameter $c$ results exhibit no radical fluctuations with respect to changing temperatures, with only a slight offset around the $31^{\circ} \mathrm{C}$ mark. To quantify the variance between the data points, the standard of deviation was calculated to be 0.1645 . The linear fit to the data set produced the following relationship:

$$
c=0.0043 \times T_{s}+1.7284
$$

where, $c$ is the shape parameter and $T_{s}$ is the measured sample temperature. From equation 4.2, it can be noted that parameter $c$ changes at a rate of $0.0043 /{ }^{\circ} \mathrm{C}$. The linear trend was not proven to be statistically significant as it yielded a regression value of 0.111 , which indicates a low correlation between the empirical data and the line of best fit. Likewise, the shape parameter $v$ exhibits noteworthy changes in the measurand as the temperature was modulated, except for its descending trend. In addition, data points demonstrated little variance with a standard deviation of 0.366. The linear fit of the following shape parameter is described by the following equation:

$$
v=-0.0055 \times T_{s}+1.2676
$$

where, $v$ is the shape and $T_{s}$ is the measured sample temperature. The descending rate of change for the shape parameter $v$ was also considerably low, which had a value of 0.0055 . The linear regression value of the line of best fit quantified at 0.336 , which implies a weak correlation between the changes in temperature and the shape parameter. The ratio of the two shape parameters also poses a similar pattern to the results of shape parameter $c$, as it is a scaled by dividing shape parameter $v$. It can be noted from the ratio and statistical analysis of the shape parameters $c$ and $v$ that there is minimal impact from changes in temperature, which has been validated with low standard of deviation quantities and a stagnant trend that can be observed in Figure 4.10 (images a and $\mathrm{b})$. As previously mentioned, the ratio $c / v$ has been related to the scatterer density of a sample in HFUS envelope statistics. Even though these envelope statistical references are still thoroughly being researched for OCT envelope statistics, the results strongly coincide with the HFUS theories. As the temperature is alternated, the scatterer density did not change as a result, and therefore displayed little to no discrepancies in the parameter as observed in the results. 
The most notable trend that was observed from the generalized gamma function was with scale parameter $a$ because it revealed a notable ascending drift with respect to increasing temperature. The results of parameter $a$ were fitted to a line of best fit, as presented in the following equation:

$$
a=0.1390 \times T_{s}+9.0504
$$

where, $a$ was the scaling parameter and $T_{s}$ was the measured sample temperature. The visual depiction of the linear trend is given in Figure 4.11.

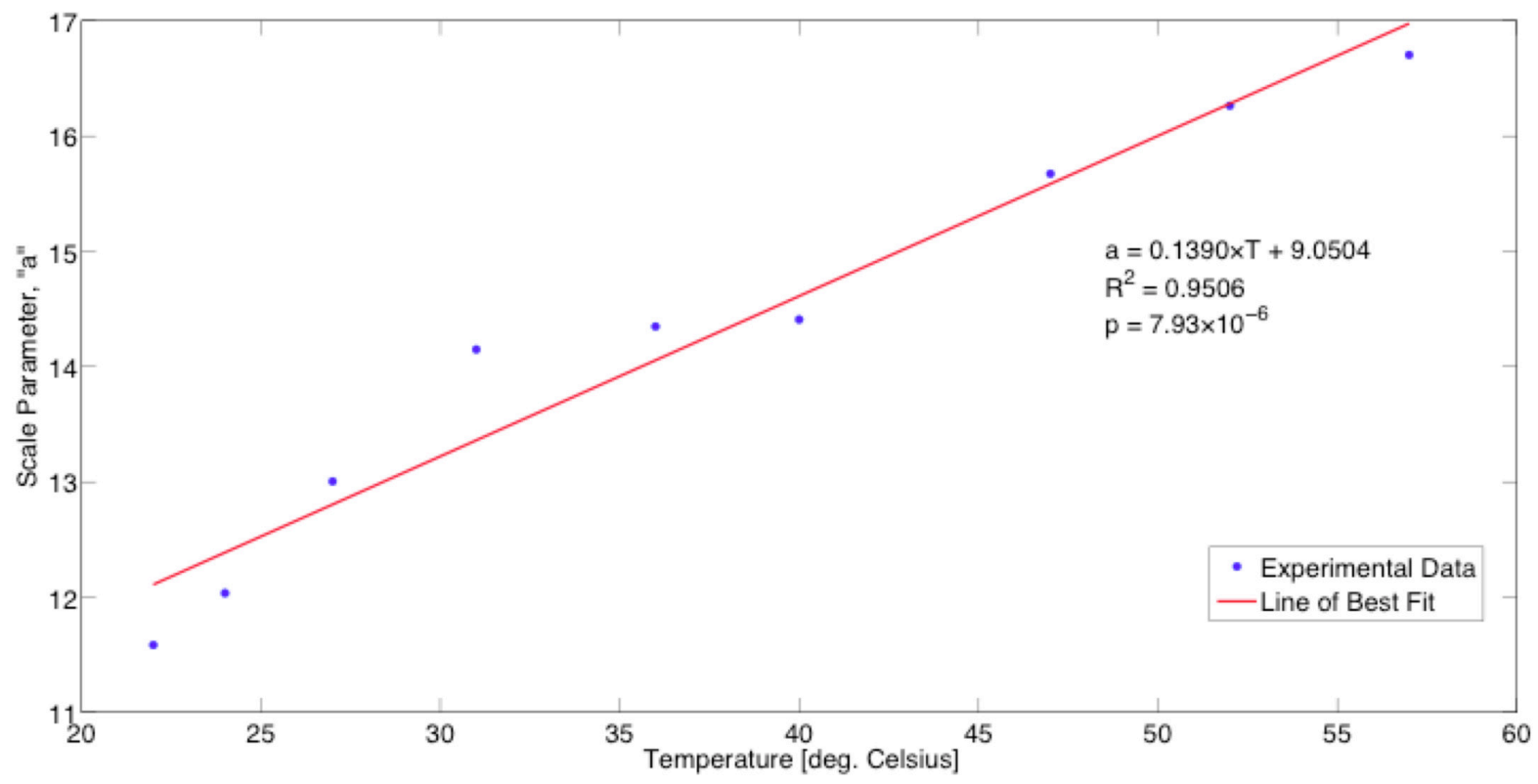

Figure 4.11: The line of best fit for the generalized gamma parameter $a$. The linear trend has a high correlation factor and is proven statistically significant with validation from the student- $t$ test [49].

The line of best fit had a regression factor of 0.9506 , which exemplifies a high correlation between the fit and the experimental data. The student t-test was performed to affirm whether the regression coefficient is coincidental or statistically valid. The results of the test provide a p-value of $7.93 \times 10^{-6}$ with a significance level of $5 \%$. A low p-value indicates that the null hypothesis of 
the scale parameter $a$ not being dependent with respect to changing temperature can be rejected. This provides confirmation that this event was not purely chance and is considered statistically significant.

Relating back to former research findings, the scale parameter $a$ is commonly associated to quantifying scatterer size [43]. The incrementing tendency of the scale parameter conveys that structural changes are occurring within the phantom with respect to changing temperature. In thermodynamics, it is common knowledge that with higher temperatures the particles experiences expansion, which in turn will increase the microspheres diameter and correspondingly also influence an increase in the scale parameter $a$. It is evident that the intensities within the OCT images are impacted by changes in temperature, however the movements of the scatterers in and out of the frame are randomized.

The linear trend observed in this trial proved its potential as a method for monitoring temperature changes in phantoms that resemble similar optical properties as biological tissue. The next step of progression is to analyze scatter sizes that are relevant to biological cell sizes, which is more ideal when relating back the findings to biological outcomes. The axial resolution of the system in air is equal to about $6.9 \mu \mathrm{m}$ and is subject to change with the refractive index of the sample. The refractive index of the tissue-mimicking phantom is equal to 1.47 and will alter the axial resolution to $10.14 \mu \mathrm{m}$ in the sample. The generalized gammas scale parameter is suggested to be a representation of scatterer size and therefore requires the OCT modality to be able to distinguish the scatterers in the image [44]. The mean diameter of scatterers is $0.75 \mu \mathrm{m}$, which indicate that OCT system cannot resolve the scatterers. In order to detect the expansion changes in the scatterers from thermal variations, scatterers larger than the axial resolution are recommendeded to further improve the efficiency of the algorithm. Golnaz et al. research on monitoring cell apoptosis using OCT envelope statistics, had used cells (AML \& PC-3) that ranged between $12.5 \mu \mathrm{m}$ and 30 $\mu \mathrm{m}$, which is well above the axial resolution of the OCT system [27]. The research was successful in tracing the structural changes in the cells using scale parameter $a$ and therefore fabricating 
phantoms with larger diameters could potentially quantify the local temperature in the microsphere phantoms. The correlation between hypo/hyperthermic influenced structural changes can then be applied in in vitro studies and progress towards an in vivo analysis.

\subsection{Chapter 4 Summary}

This chapter introduced the statistical basis of monitoring induced thermal changes in tissuemimicking phantoms. It was determined that the generalized gamma PDF was the most apt distribution to represent the statistical envelope of OCT images of the tissue-mimicking phantoms; as it produced the lowest correlation factor based on the KS test. Building on the generalized gamma function, the parameters were observed with respect to modulated temperatures. The microsphere phantom exhibited a linear trend with the scale parameter $a$, as the temperature was incremented. The results show promise in producing a viable metric to quantifying temperature in local regions of thermal administration. Monitoring the thermal response during thermal ablative procedures could lead to a better understanding of the biological outcomes of the tumors, which could further the development of more successful therapy procedures. Advancing the potential of OCT envelope statistics requires further analysis and a comprehensive clinical study. 


\section{Chapter 5}

\section{Conclusion}

\subsection{Summary}

The main motivation of this thesis was to acknowledge the limitations clinicians face during thermal ablative procedures and to constitute a potential solution with the use of optics. OCT has demonstrated potential in establishing metrics to track the progression of cancer therapy outcomes, and in this thesis we have proposed a method to monitor the local temperature of tumors for thermal ablative procedures.

Overall, the main objective of this thesis was to trace local thermal changes in tissue-mimicking phantoms by analyzing the envelope statistics of structural OCT images. In addition, the groundwork was established for building a multi-functional probe that is capable of measuring the local temperature with optical sensors and OCT imaging.

Chapter 1 provides the incentive behind the scope of the project, a general overview of current thermal ablative methodologies and proposed modes of temperature monitoring. Moreover, the benefits of OCT were also listed, which provided the basis as to why OCT is a highly attractive modality for the purpose of this research.

Chapter 2 discusses the theory behind OCT envelope statistics and the optical sensor, FBG. The application of the theory with respect to thermal sensing is further analyzed, along with prior 
research history within the domain of US and OCT envelope statistics (which are analogous).

Chapter 3 dealt with the development of an FBG-thermal sensing probe and a standardization curve to examine the thermal feedback of the sensors. The sensor has shown prospective in the application of medical catheters due to its beneficial characteristics such as its robustness and small dimensions.

Lastly, chapter 4 outlines the implementation of an OCT envelope statistics algorithm for monitoring thermal variations. The generalized gamma functions parameters were proven to be statistically effective in quantifying the local temperature in the microsphere suspension fluid phantom that resembled the optical properties of biological tissue.

This optical thermal sensing toolkit of techniques provided in this thesis provides the fundamental groundwork that can aid in answering some biological doubts for thermal ablative therapies. Furthermore, such knowledge can help to increase the efficiency and success rates of procedures of this nature, aiding in the progression of improving the quality of life for patients who undergo such therapy options. 


\section{References}

[1] Canadian Cancer Statistics Special topic : Liver cancer. Technical report, Canadian Cancer Society, Toronto, 2013.

[2] Loraine D Marrett, Prithwish De Mhsc, Parisa Airia, and Dagny Dryer. Cancer in Canada in 2008. Canadian Medical Association Journal, 179(11):1163-1170, 2008.

[3] L Kachuri, P De, L F Ellison, and R Semenciw. Cancer incidence, mortality and survival trends in Canada, 1970-2007. Chronic diseases and injuries in Canada, 33(2):69-80, March 2013.

[4] James E. Kennedy. High-intensity focused ultrasound in the treatment of solid tumours. Nature Reviews Cancer, 5(4):321-7, June 2005.

[5] J E Kennedy. High intensity focused ultrasound: surgery of the future? British Journal of Radiology, 76(909):590-599, September 2003.

[6] SA Curley, Francesco Izzo, Paolo Delrio, and LM Ellis. Radiofrequency ablation of unresectable primary and metastatic hepatic malignancies: results in 123 patients. Annals of Surgery, 230(1):1-8, 1999.

[7] D E Dupuy, R Hong, B Oliver, and S N Goldberg. Radiofrequency ablation of spinal tumors: temperature distribution in the spinal canal. American journal of roentgenology, 175(5):1263-6, November 2000.

[8] Bharat Gowardhan and Damien Greene. Cryotherapy for the prostate: an in vitro and clinical 
study of two new developments; advanced cryoneedles and a temperature monitoring system. BJU international, 100(2):295-302, August 2007.

[9] AC Steger and WR Lees. Interstitial laser hyperthermia: a new approach to local destruction of tumours. BMJ: British Medical ..., 299:362-5, 1989.

[10] Katrina F Chu and Damian E Dupuy. Thermal ablation of tumours: biological mechanisms and advances in therapy. Nature Reviews Cancer, 14(3):199-208, March 2014.

[11] Chris J Diederich. Thermal ablation and high-temperature thermal therapy: overview of technology and clinical implementation. International journal of hyperthermia : the official journal of European Society for Hyperthermic Oncology, North American Hyperthermia Group, 21(8):745-53, December 2005.

[12] YF Zhou. High intensity focused ultrasound in clinical tumor ablation. World Journal of Clinical Oncology, 2(1):8-27, 2011.

[13] Muneeb Ahmed, Christopher L Brace, Fred T Lee, and S Nahum Goldberg. Principles of and Advances in Percutaneous Ablation. Radiology, 258(2):351-369, 2011.

[14] Mehrdad Nikfarjam, Vijayaragavan Muralidharan, and Christopher Christophi. Mechanisms of focal heat destruction of liver tumors. The Journal of surgical research, 127(2):208-23, August 2005.

[15] Michael S Sabel. Cryo-immunology: a review of the literature and proposed mechanisms for stimulatory versus suppressive immune responses. Cryobiology, 58(1):1-11, March 2009.

[16] Tom Mala. Cryoablation of liver tumours - a review of mechanisms, techniques and clinical outcome. Minimally invasive therapy \& allied technologies : MITAT : official journal of the Society for Minimally Invasive Therapy, 15(1):9-17, January 2006.

[17] T Mala, E Samset, L Aurdal, I Gladhaug, B Edwin, and O Sø reide. Magnetic resonance imaging-estimated three-dimensional temperature distribution in liver cryolesions: a study of 
cryolesion characteristics assumed necessary for tumor ablation. Cryobiology, 43(3):268-75, November 2001.

[18] Joseph L Chin, Darwin Lim, and Mazen Abdelhady. Review of Primary and Salvage Cryoablation. pages 231-237, 2007.

[19] Dan Theodorescu. Cancer Cryotherapy: Evolution and Biology. Reviews in Urology, 6(4):919, 2004.

[20] I. Jovanovic, A. Hormati, P. Littrup, N. Duric, O. Rama, and M. Vetterli. Temperature monitoring during tissue freezing using ultrasound speed measurements. In Stephen A. McAleavey and Jan D'hooge, editors, Medical Imaging 2009: Ultrasonic Imaging and Signal Processing, volume 7265, pages 72650Q-72650Q-8, February 2009.

[21] Eigil Samset. Temperature mapping of thermal ablation using MRI. Minimally invasive therapy \& allied technologies : MITAT : official journal of the Society for Minimally Invasive Therapy, 15(1):36-41, January 2006.

[22] Adrian Mariampillai. Development of a high resolution microvascular imaging toolkit for optical coherence tomography. Phd thesis, University of Toronto, 2011.

[23] Beau A Standish, Kenneth K C Lee, Xiao Jin, Adrian Mariampillai, Nigel R Munce, Michael F G Wood, Brian C Wilson, I Alex Vitkin, and Victor X D Yang. Interstitial Doppler optical coherence tomography as a local tumor necrosis predictor in photodynamic therapy of prostatic carcinoma: an in vivo study. Cancer research, 68(23):9987-95, December 2008.

[24] AF Fercher and W Drexler. Optical coherence tomography-principles and applications. Reports on Progress in Physics, 66:239-303, 2003.

[25] Beau A. Standish, Xiao Jin, Jurek Smolen, Adrian Mariampillai, Nigel R Munce, Brian C Wilson, I Alex Vitkin, and Victor X D Yang. Interstitial Doppler optical coherence tomography monitors microvascular changes during photodynamic therapy in a Dunning prostate model under varying treatment conditions. Journal of biomedical optics, 12(3):034022, 2014. 
[26] Adrian Mariampillai, Beau a Standish, Eduardo H Moriyama, Mamta Khurana, Nigel R Munce, Michael K Leung, James Jiang, Alex Cable, Brian C Wilson, I Alex Vitkin, and Victor X D Yang. Speckle variance detection of microvasculature using swept-source optical coherence tomography. Optics letters, 33(13):1530-2, July 2008.

[27] Golnaz Farhat, Victor X D Yang, Gregory J Czarnota, and Michael C Kolios. Detecting cell death with optical coherence tomography and envelope statistics. Journal of biomedical optics, 16(2):026017, February 2011.

[28] Joseph M. Schmitt. Optical coherence tomography (OCT): a review. IEEE Journal of Selected Topics in Quantum Electronics, 5(4):1205-1215, 1999.

[29] Bowei Zhang. Optical High Temperature Sensor Based on Fiber Bragg Grating. PhD thesis, Concordia University, 2007.

[30] Y. Rao, D.J. Webb, D.A. Jackson, and I. Bennion. In-fiber Bragg-grating temperature sensor system for medical applications. Journal of Lightwave Technology, 15(5):779-785, May 1997.

[31] W W Morey, J R Dunphy, and G Meltz. Multiplexing fiber Bragg grating sensors. In Distributed and Multiplexed Fiber Optic Sensors, volume 1586, pages 216-224, 1992.

[32] Subaagari Seevaratnam, Mashal Farid, Golnaz Farhat, and Beau A. Standish. Analyzing effects of temperature on tissue equivalent phantoms using fiber Bragg gratings and optical coherence tomography. In Pavel Cheben, Jens Schmid, Caroline Boudoux, Lawrence R. Chen, André Delâge, Siegfried Janz, Raman Kashyap, David J. Lockwood, Hans-Peter Loock, and Zetian Mi, editors, Photonics North 2013, volume 8915, page 891504, Ottawa, October 2013.

[33] Chen Wei-gen, Liu Jun, Wang You-yuan, Liang Liu-ming, Zhao Jian-bao, and Yue Yanfeng. The Measuring Method for Internal Temperature of Power Transformer Based on FBG Sensors. In 2008 International Conference on High Voltage Engineering and Application, pages 672-676. Ieee, November 2008. 
[34] A.D. Kersey, M.A. Davis, H.J. Patrick, M. LeBlanc, K.P. Koo, C.G. Askins, M.A. Putnam, and E.J. Friebele. Fiber grating sensors. Journal of Lightwave Technology, 15(8):1442-1463, 1997.

[35] Jieyan Li, Dongsheng Zhang, Xiaoyan Wen, and Litong Li. Fabrication and Temperature Characteristics of Thermal Regenerated Fiber Bragg Grating. In 2012 Symposium on Photonics and Optoelectronics, volume 2, pages 1-4. Ieee, May 2012.

[36] Byoungho Lee. Review of the present status of optical fiber sensors. Optical Fiber Technology, 9(2):57-79, April 2003.

[37] Andreas Othonos, Kyriacos Kalli, David Pureur, and Alain Mugnier. Fibre Bragg Gratings. In Wavelength Filters in Fibre Optics, chapter 5, pages 189-269. Springer, Germany, 2006.

[38] A S Tunis, G J Czarnota, A Giles, M D Sherar, J W Hunt, and M C Kolios. Monitoring structural changes in cells with high-frequency ultrasound signal statistics. Ultrasound in medicine \& biology, 31(8):1041-9, August 2005.

[39] P M Shankar, J M Reid, H Ortega, C W Piccoli, and B B Goldberg. Use of non-Rayleigh statistics for the identification of tumors in ultrasonic B-scans of the breast. IEEE transactions on medical imaging, 12(4):687-92, January 1993.

[40] Xiaohui Hao, Charles J Bruce, Cristina Pislaru, and James F Greenleaf. Segmenting highfrequency intracardiac ultrasound images of myocardium into infarcted, ischemic, and normal regions. IEEE transactions on medical imaging, 20(12):1373-83, December 2001.

[41] P M Shankar. A model for ultrasonic scattering from tissues based on the $\mathrm{K}$ distribution. Physics in medicine and biology, 40(10):1633-49, October 1995.

[42] P Mohana Shankar. A compound scattering pdf for the ultrasonic echo envelope and its relationship to $\mathrm{K}$ and Nakagami distributions. IEEE transactions on ultrasonics, ferroelectrics, and frequency control, 50(3):339-43, March 2003. 
[43] Balasundar I Raju and Mandayam A Srinivasan. Statistics of envelope of high-frequency ultrasonic backscatter from human skin in vivo. IEEE transactions on ultrasonics, ferroelectrics, and frequency control, 49(7):871-82, July 2002.

[44] A.S. Tunis, R.E. Baddour, G.J. Czarnota, A Giles, A.E. Worthington, M.D. Sherar, and M.C. Kolios. Using high frequency ultrasound envelope statistics to determine scatterer number density in dilute cell solutions. In IEEE Ultrasonics Symposium, 2005., volume 2, pages 878-881. IEEE, 2005.

[45] P M Shankar. Ultrasonic tissue characterization using a generalized Nakagami model. IEEE transactions on ultrasonics, ferroelectrics, and frequency control, 48(6):1716-20, November 2001.

[46] V. Dutt and J. F. Greenleaf. Ultrasound Echo Envelope Analysis Using a Homodyned K Distribution Signal Model. Ultrasonic Imaging, 16(4):265-287, October 1994.

[47] EW Stacy. A generalization of the gamma distribution. The Annals of Mathematical Statistics, 33(3):1187-1192, 1962.

[48] W J Conover. Practical Nonparametric Statistics. John Wiley and Sons, Incorporated, New York, 2 edition, 1980.

[49] Subaagari Seevaratnam, Amitpal Bains, Mashal Farid, Golnaz Farhat, Michael Kolios, and Beau A. Standish. Quantifying temperature changes in tissue-mimicking fluid phantoms using optical coherence tomography and envelope statistics. In Israel Gannot, editor, Photonics West 2014, volume 8938, page 89380R, February 2014.

[50] Vesselina Tsenova and EV Stoykova. Refractive index measurement in human tissue samples. In Peter A. Atanasov, Alexander A. Serafetinides, and Ivan N. Kolev, editors, 12th International School on Quantum Electronics: Laser Physics and Applications, volume 5226, pages 413-417, November 2003. 
[51] Discover the origins of some of the world's most consistently pure products... synthetic glycerine products. Technical report, The DOW Chemical Company, Michigan, Mexico City and Horgen, 1999.

[52] Stefka Nikolova Kasarova, Nina Georgieva Sultanova, Christo Dimitrov Ivanov, and Ivan Dechev Nikolov. Analysis of the dispersion of optical plastic materials. Optical Materials, 29(11):1481-1490, July 2007.

[53] Golnaz Farhat, Adrian Mariampillai, Victor X. D. Yang, Gregory J. Czarnota, and Michael C. Kolios. Optical coherence tomography speckle decorrelation for detecting cell death. SPIE BiOS, 7907:790710-790710-10, February 2011. 


\section{Appendix A}

\section{Additional Work: Speckle Decorrelation}

\section{A.0.1 Speckle Decorrelation Measurements Potential to Quantify Local Tem- peratures}

Brownian motion is defined as the random movement of microscopic particles suspended in a liquid solution. The event transpires due to the suspended particles colliding with its surrounding medium. Previous research has proven that Brownian motion can be measured with dynamic light scattering or also known as photon correlation spectroscopy, which is measuring the backscattered light (via detector) from a light beam that is aimed towards a scattering medium [53]. The dynamic light scattering measurement can be performed with OCT and also has the potential to measure localized temperature in a phantom [53]. Quantifying the decorrelation time of speckle at each temperature setting can provide viable information to understand the local temperature in a phantom. The phantom that was finalized for the envelope statistics was also convenient to use for measuring speckle decorrelation. The speckle decorrelation measurement was acquired by monitoring the speckle fluctuation in the OCT images of the monodisperse microsphere phantom as a function of time. Such information is obtained by acquiring a collection of OCT frames of phantom and then from a standard ROI (amongst all frames) a pixel of interest is chosen. The intensity of the OCT frames are plotted with respect to the duration of time the phantom images were recorded, which is depicted in Figure A.1. 


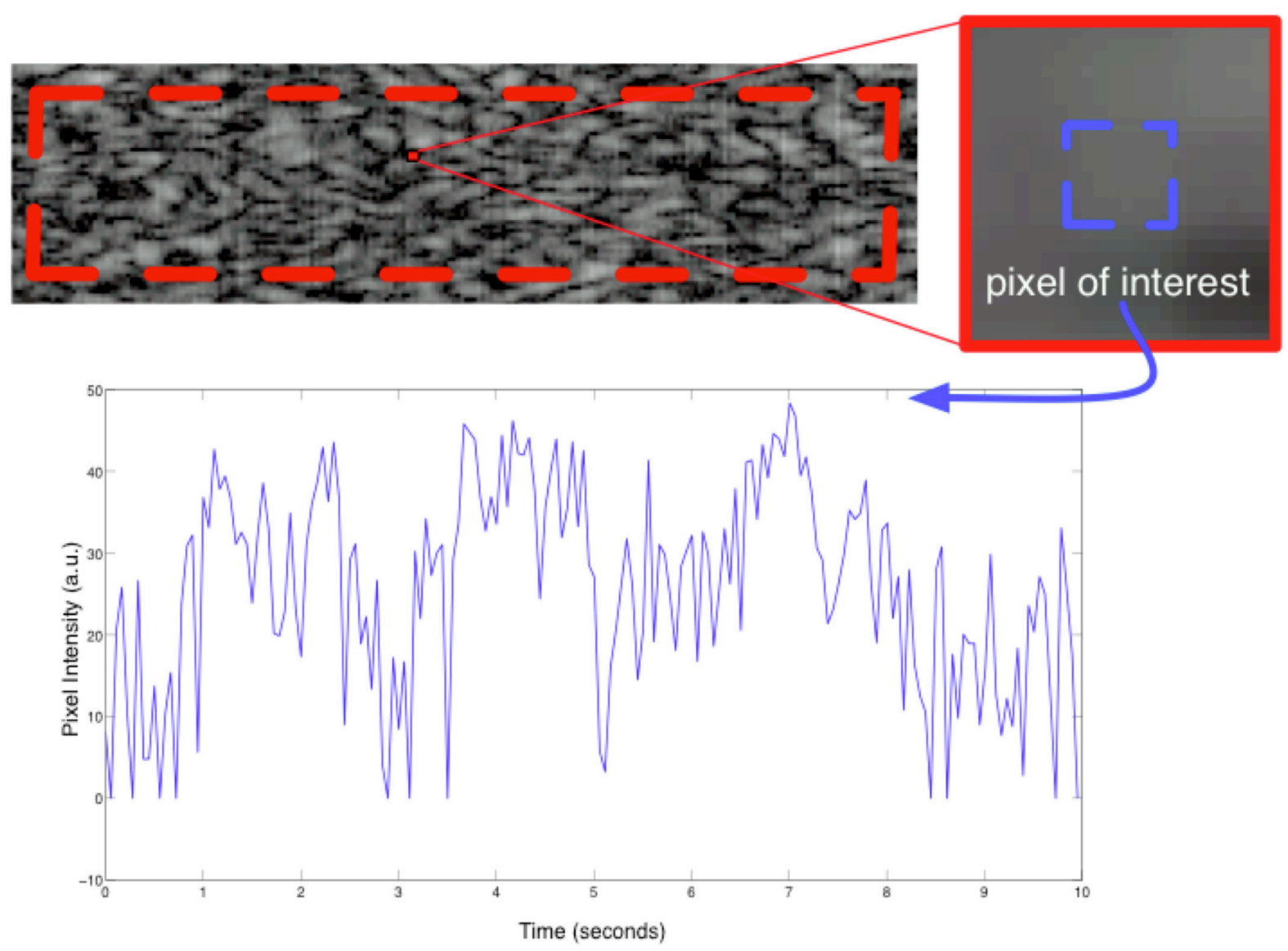

Figure A.1: Portrayal of the intensity fluctuation in the speckle and the corresponding time signal [49]. The dashed red box is the ROI, and the blue box is the pixel of interest. The time-dependent intensity signal looks similar to a fringe signal. 
In order to measure the decorrelation time, the autocorrelation function was applied on the time-dependent intensity signal. The autocorrelation function of the monodisperse microsphere phantom which, undergoes Brownian motion can be described using an exponentially decaying function as shown in equation A.1 [49]:

$$
A(\tau)=e^{-2 q^{2} D_{B} t}
$$

where, $q$ is the wave vector notation, $D_{B}$ is the diffusion coefficient, $t$ is time and $\tau$ is the measured time constant. The wave vector and the diffusion coefficients are represented by the following expressions [49]:

$$
q=\frac{4 \pi}{\lambda}
$$

and

$$
D_{B}=\frac{\kappa_{B} T}{6 \pi n r}
$$

where, $\lambda$ is the wavelength of incident, $\kappa_{B}$ is the Boltzmanns constant, $T$ is the absolute temperature, $n$ is the viscosity of the medium (glycerol) and $r$ is the particle radius. The decorrelation time can then be determined with the following equation [49]:

$$
\tau=\frac{1}{2 q^{2} D_{B}}
$$

The empirical data from the OCT frames will be used to acquire an autocorrelation function, which is then comparatively examined with an exponential fit that can will provide the necessary information to measure the decorrelation time using equation A.4.

The preliminary results of the decorrelation measurements have shown promising outcomes, where the experimental data revealed an exponential decay and its exponential fit matched well. Figure A.2 is an example of the autocorrelation function at $36^{\circ} \mathrm{C}$, which was acquired using the in-house SS-OCT system. For this example, the empirical data measurement of decorrelation time 
was $246.4419 \mathrm{~ms}$, which did not comply with the theoretical value of 1.1453 seconds. The OCT system was only capable of acquiring 18 frames per second, which provides limited information to obtain accurate results. Prior research in measuring speckle decorrelation using OCT data by Golnaz et al. had used a system with a frame rate of $166 \mathrm{~Hz}$, which is significantly higher than the rate utilized in this research [53]. For future reference, it is key to note that to increase the imaging frame rate one must limit the field of view while imaging. Therefore concentrating the focus in a high signal to noise region would be more idealistic for measuring the decorrelation time.

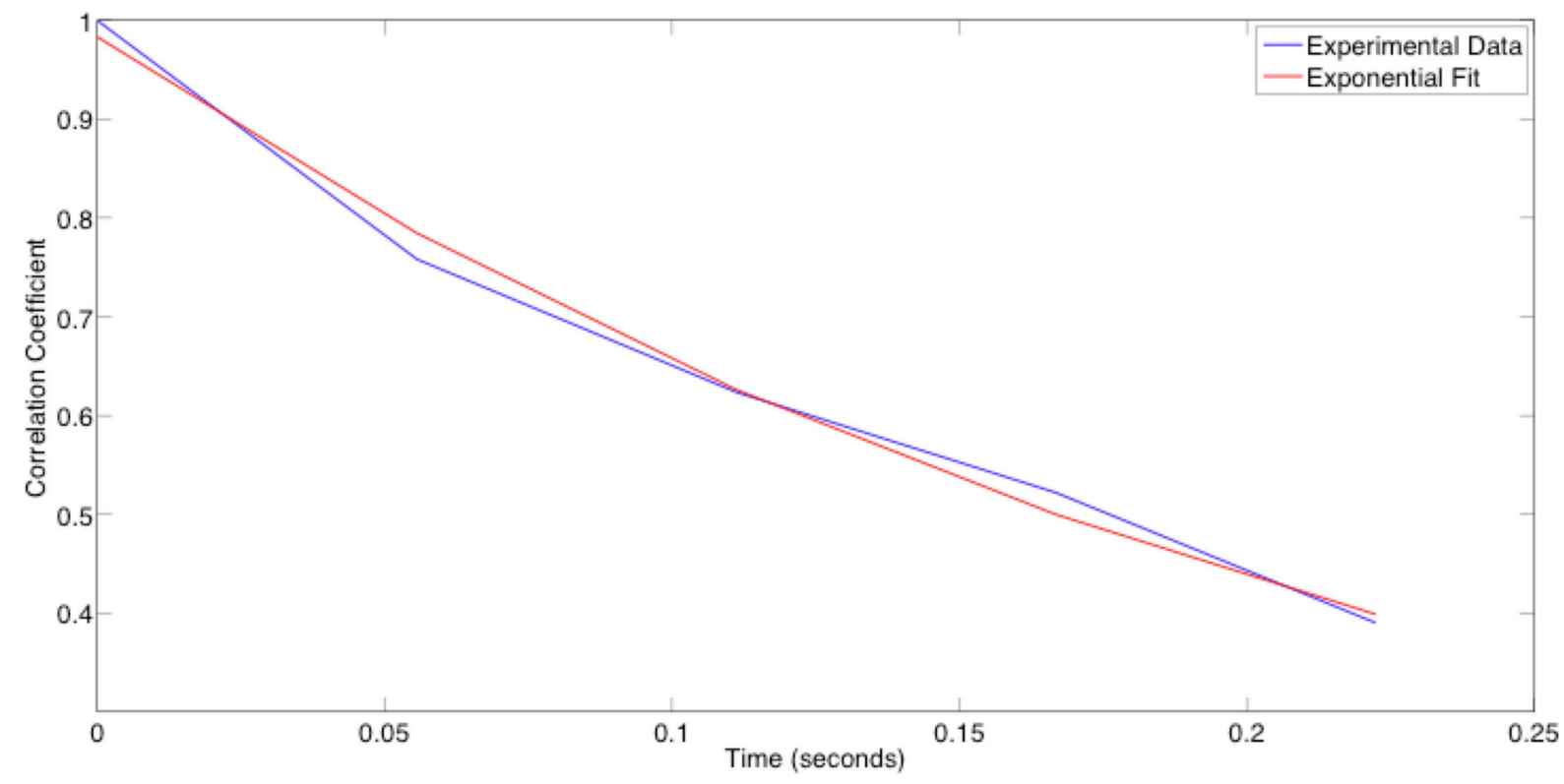

Figure A.2: The autocorrelation function of the intensity time signal at a sample temperature of 36C of a microsphere suspension fluid phantom [49].

Measuring the decorrelation time based on speckle is a difficult task, as the scatterers are easily influenced by motion artifacts. The slightest vibration or impact to the experimental setup can distort the speckle intensity because it can alter the movement of the scatterers. However, the decorrelation time of a monodisperse microsphere phantom can be easily modeled with a mathematical representation, which can be a reference to improving the procedure of measuring speckle decorrelation with respect to temperature. Overall, there are promising possibilities with speckle 
decorrelation and envelope statistics methods to measure local temperature during thermal ablation. 


\section{Appendix B}

\section{Research Contributions}

The following written conference proceedings, oral presentations and poster presentations were produced from the research conducted in this thesis work:

\section{Conferences}

- S. Seevaratnam, M. Farid, G. Farhat, and B. A. Standish, "Analyzing effects of temperature on tissue equivalent phantoms using fiber Bragg gratings and optical coherence tomography," in Photonics North 2013, 2013, vol. 8915, p. 891504.

- S. Seevaratnam, A. Bains, M. Farid, G. Farhat, M. Kolios, and B. A. Standish, "Quantifying temperature changes in tissue-mimicking fluid phantoms using optical coherence tomography and envelope statistics," in Photonics West 2014, 2014, vol. 8938, p. 89380R.

\section{Oral Presentations}

- S. Seevaratnam, M. Farid, G. Farhat, M. C. Kolios and B. A. Standish, (February 2014) "Optical Coherence Tomography Envelope Statistics Methodology to Assess Temperature Changes in Tissue Mimicking Fluids." at The International Society for Optical Engineering Photonics West 2014. San Francisco, CA. 


\section{Poster Presentations}

- S. Seevaratnam and B. A. Standish, (November 2012) "Temperature as a Potential Contrast Mechanism for Optical Coherence Tomography," at Ryerson Research Symposium 2012. Toronto, ON.

- S. Seevaratnam, M. Farid and B. A. Standish, (June 2013) "Optical Coherence Tomography Envelope Statistics to Detect Changes in Temperatures Tissue Mimicking Phantom," Biomedical Engineering and Sciences Technology (BEST) Research Symposium 2013 (St. Mikes Hospital). Toronto, ON.

- S. Seevaratnam, M. Farid, G. Farhat, M. C. Kolios and B. A. Standish, (November 2013) "Using Optical Coherence Tomography Speckle Decorrelation to Measure the Local Temperature in a Microbead Tissue-Mimicking Suspension Phantom,” Ryerson Research Symposium 2013. Toronto, ON.

- S. Seevaratnam, M. Farid, G. Farhat, M. C. Kolios and B. A. Standish, (March 2014) “Quantifying the Relationship between Optical Coherence Tomography Speckle Decorrelation Time and Temperature in Tissue-Mimicking Suspension Phantoms," at Imaging Network Ontario Syposium 2014. Toronto, ON. 